Original reference:

Perez-Osorio J, Abubshait A, \& Wykowska A. (2021). Irrelevant Robot Signals in a Categorization Task Induce Cognitive Conflict in Performance, Eye Trajectories, the N2 Component of the EEG Signal, and Frontal Theta Oscillations. Journal of Cognitive Neuroscience. Dec 6;34(1):108-126. doi: 10.1162/jocn_a_01786. PMID: 34705044 .

\title{
Irrelevant Robot Signals in a Categorization Task Induce Cognitive Conflict in Performance, Eye trajectories, the N2 component of the EEG, and Frontal Theta Oscillations
}

Jairo Perez-Osorio*, Abdulaziz Abubshait*, \& Agnieszka Wykowska Istituto Italiano di Tecnologia, Social Cognition in Human-Robot Interaction

Keywords: Visuospatial signals, cognitive conflict, eye movement trajectories, EEG/ERPs, theta oscillations

\section{${ }^{*}$ Equal contribution}

Corresponding author: Agnieszka Wykowska: agnieszka.wykowska@iit.it 


\begin{abstract}
Understanding others' nonverbal behavior is essential for social interaction, as it allows, among others, to infer mental states. While gaze communication, a well-established nonverbal social behavior, has shown its importance in inferring others' mental states, not much is known about the effects of irrelevant gaze signals on cognitive conflict markers during collaborative settings. Here, participants completed a categorization task where they categorized objects based on their color while observing images of a robot. On each trial, participants observed the robot iCub grasping an object from a table and offering it to them to simulate a handover. Once the robot "moved" the object forward, participants were asked to categorize the object according to its color. Before participants were allowed to respond, the robot made a lateral head/gaze shift. The gaze shifts were either congruent or incongruent with the object's color. We expected that incongruent head-cues would induce more errors (Study 1), would be associated with more curvature in eye-tracking trajectories (Study 2), and induce larger amplitude in electrophysiological markers of cognitive conflict (Study 3). Results of the three studies show more oculomotor interference as measured in error rates (Study 1), larger curvatures eye-tracking trajectories (Study 2), and higher amplitudes of the N2 eventrelated potential (ERP) of the EEG signals as well as higher Event-Related Spectral Perturbation (ERSP) amplitudes (Study 3) for incongruent trials compared to congruent trials. Our findings reveal that behavioral, ocular and electrophysiological markers can index the influence of irrelevant signals during goal-oriented tasks.
\end{abstract}




\section{Introduction}

Understanding others' mental states (e.g., intentions, thoughts, feelings, and emotions) is critical for social interaction. Mental states, however, are covert in nature, and people have to attend to others' overt social signals in behavior to infer and predict their mental states (Adolphs, 2003; Gallagher \& Frith, 2003). For example, others' gaze direction reveals their locus of attention and highlights potentially relevant objects, events and locations. Following gaze can also provide hints to facilitate coordinated actions between people to reach desired goals (Frischen et al., 2007). Nevertheless, in certain situations, these social signals can provide conflicting information to achieve a desired goal. Moreover, further research is required to understand how conflicting signals from artificial agents can influence cognitive processes in collaborative scenarios (i.e., a robot assisting a human in a warehouse or greeting clients at a front desk). Therefore, it is imperative to systematically examine the effect of conflicting "social" signals (e.g., gaze direction) on people's behavior during goal-oriented tasks that demand cognitive control.

Gaze following has been widely investigated in the laboratory using the gazecueing task (Friesen \& Kingstone, 1998). In this task, a straight-ahead-looking face is presented on a screen. Then, the face would shift its head/gaze towards a location in space (i.e., a gaze-cue). Next, a target appears at either the same (i.e., valid cue) or a different location (i.e., invalid cue). Observers generally respond faster to validly cued targets than invalidly cued targets (i.e., gaze cueing effect). Studies propose that gaze following is reflexive and domain-general (Friesen \& Kingstone, 1998; Quadflieg et al., 2004). These findings mirror Posner cueing effects with endogenous cues suggesting that spatial cueing directs attentional resources regardless of the cue type (i.e., faces, 
eyes, or arrows) (Brignani et al., 2009; Callejas et al., 2014). Nevertheless, an integrative framework (Kuhn et al., 2018) and mounting evidence suggest that the social relevance of a face can exert top-down effects and enhance the gaze-cueing effect (Ishikawa et al., 2021; Tipples, 2006; Fox et al., 2007; Bonifacci et al., 2008; Gobel et al., 2017; Graham et al., 2010; Kawai, 2011; Hungr \& Hunt, 2012; Perez-Osorio et al., 2015, 2017; Süßenbach and Schönbrodt, 2014; Wykowska et al., 2014; Cazzato et al., 2015; Dalmaso et al., 2015, 2020; Marotta et al., 2012; Abubshait \& Wiese, 2017; Abubshait et al., 2020; see Capozzi \& Ristic, 2020 for review). For example, when observers ascribe mental states to a gazer, their gaze-cueing effect is enhanced (Kuhn et al., 2018; Teufel et al., 2010; Cole et al., 2015). Collectively, this work suggests that following others' gaze is a cost-effective way to obtain relevant information about the environment's current and future state. Still, the meaning carried by those social signals depends on how observers read the context and the gazers involved.

The ability to read and understand social signals extends to artificial agents and not just human interaction partners. Studies supported the idea that humanoid robots can elicit attention shifts to the same degree as humans (e.g., Chaminade \& Okka, 2013; see Chevalier et al., 2020, Wykowska et al., 2016 and Wiese et al., 2017, Wykowska, 2020 for review). Specifically, observers were able to exert top-down modulation of gaze-cueing when interacting with a robot endowed with characteristics that increased its social relevance (Abubshait \& Wiese, 2017; Wiese et al., 2018; Wiese et al., 2012; Wykowska et al., 2014). These findings are also extended in more ecologically valid paradigms that measure gaze-cueing in real Human-Robot Interaction settings (Abubshait \& Wykowska, 2020; Kompatsiari et al., 2017; 2018). It has also been shown that robots can use social signals in more complex scenarios (i.e., head/gaze cue) to 
effectively convey messages during storytelling (Mutlu et al., 2006), to facilitate joint actions (Boucher et al., 2012), improve the perception of competence (Huang \& Thomaz, 2011) and provide hints to solve tasks (Mutlu et al., 2006). EEG studies also mirror behavioral findings by showing that attentional orienting during the observation of gaze/head shifts in humanoid robots elicit similar electrophysiological responses as with human faces (e.g., earlier and more pronounced N1 for valid relative to invalid cue trials (Kompatsiari et al., 2018)). In summary, research has shown that people seem to interpret signals exhibited by humanoid robots socially, suggesting that humans recruit similar cognitive mechanisms to interact with artificial agents as they do with humans (Wykowska, 2020). However, it remains to be answered whether this is due to perceiving the robots as social agents or because the recruited cognitive mechanisms are of "general-purpose" type rather than specifically social.

One of the open questions in human-robot interaction research is the interaction between cognitive control and attentional orienting induced by a socially salient signal in a bottom-up manner. For example, is it possible to cognitively control (and thus suppress) irrelevant salient (social) signals from a humanoid robot? The present study aims to evaluate the impact of conflicting robot social signals on performance reflected in behavioral and neural markers during a task requiring cognitive control. However, our study does not aim to answer whether the cognitive mechanisms evoked by the signals exhibited by the robot are essentially social or somewhat of general purpose. Instead, the aim is to understand whether endowing artificial agents with specific signals (e.g., directional gaze) that are irrelevant to the task at hand might actually be detrimental to the task. This would have far-reaching implications for cognitive control research and application areas in robot design and human factors. 


\section{Cognitive control in the brain}

Humans exist in continuously changing environments and need to keep track of internal goals and objectives. For example, when driving, watching a film, deciding which option to choose on a menu, or trying to look where someone is pointing, goals and actions need to be maintained while distractors need to be suppressed. Action goals remain active due to cognitive control mechanisms that monitor and detect conflict in information processing and prevent deviations from the goal's direction (Botvinick et al., 2001; Yeung, 2014). In the laboratory, cognitive control has been studied using paradigms that demand the processing of conflicting information and/or selection (or inhibition) of responses. These experimental designs induce a conflict between taskrelevant or -irrelevant stimulus features (e.g., the flanker task; Eriksen \& Eriksen, 1974) or between stimulus-response associations (e.g., Simon task; Simon \& Wolf, 1963). Typically, incongruent conditions produce longer response times and higher error rates, reflecting the high demand for cognitive resources and more cognitive conflict (Botvinick et al., 2001; Yeung, 2014).

Studies have shown that the brain continuously monitors and evaluates any possible conflict between expected outcomes and actual outcomes from sensory inputs to maintain cognitive control (Botvinick et al., 2001; Botvinick, Cohen \& Carter, 2004; Kerns et al., 2004; Yeung, 2014). The ACC has been identified as a possible source of the electro-cortical markers of cognitive control/outcome monitoring. (Bocquillon et al., 2014; Yeung et al., 2004; Botvinick et al., 2004). These markers related to monitoring, conflict detection, and interference are divided into stimulus processing (i.e., mismatch N2), response selection/inhibition (i.e., control N2), and feedback-related activity (i.e., FRN) and are observed in the $200-400 \mathrm{~ms}$ time window over frontocentral electrodes 
(see Folstein \& Van Petten, 2008, for a review) post-stimulus or feedback, respectively. Typically, larger amplitudes of this component signify more conflict, interference, or expectation mismatch. These conflict-related N2 components are also observed during NoGo trials in Go/NoGo tasks (Nieuwenhuis et al., 2003), during incongruent trials in the flanker task (Kopp et al., 1996; Heil et al., 2000), and the Simon task (Leuthold, 2011).

Conflict-related components are also present in oscillatory measurements of EEG. Several studies have identified slow frequency theta oscillations $(4-8 \mathrm{~Hz})$ over the midfrontal cortex as an index for cognitive conflict (Cohen \& Donner, 2013; Cavanagh, Cohen, \& Allen, 2009; Cavanagh \& Frank, 2014; Cohen \& Cavanagh, 2011; Sauseng et al., 2010). Specifically, changes in Theta power related to performance monitoring have been reported in Go/NoGo paradigms (Yamanaka \& Yamamoto, 2009), task switching paradigms (Sauseng et al., 2006), goal conflicts (Moore et al., 2006), and rule violation (Tzur \& Berger, 2007). This activity is associated with increased power in transient bursts, as quick as $300 \mathrm{~ms}$, and sustained activity that lasts 10 seconds (Mitchell, McNaughton, Flanagan, \& Kirk, 2008). While transient bursts correspond to reactive adjustment control (working memory, inhibiting a response, detecting errors), sustained theta coherence between frontal and posterior sites relates to updating and maintaining task goals. Theta oscillations may facilitate adjusting to complex tasks, allocating cognitive resources according to task demands using a common mechanism that allows dynamic communication between task-relevant cortical regions (Voytek et al., 2015). 


\section{Cognitive conflict and social signals}

While studies investigated how cognitive conflict can modulate attention orienting to social signals via processing emotional faces (Pecchinenda \& Petrucci, 2016), by inducing cognitive demands (Bobak \& Langton, 2015), or the processing of categorically ambiguous faces (Abubshait et al., 2020; Wiese et al., 2019), few studies investigated the effects of cognitive control when observing conflicting social signals. For instance, Zaki et al. (2010) measured behavioral and neural responses during the observation of conflicting verbal and nonverbal social signals (e.g., showing a happy face and the sentence "my dog died"). This study found similar neural responses as in nonsocial cognitive conflict experiments with the addition of activation from social brain structures. This suggests that specific brain structures are required to handle social cognitive conflict (Zaki et al., 2010).

Other studies have compared the difference between conflicting information from eyes and arrows and evaluated what characterizes these spatial cues in terms of cognitive conflict. For instance, Marotta and colleagues $(2018 ; 2019)$ found that incongruent spatial cues from eyes and arrows indeed generate cognitive conflict (i.e., eyes/arrow indicate left, correct response right), as reflected by slower RTs and increased brain activity relative to congruent signals. Interestingly, eyes elicited opposite congruency effects compared to arrows (faster responses to incongruent relative to congruent cues). Analysis of the neural correlates (Marotta et al., 2019) revealed that conflicting information from eyes and arrows elicits identical brain activity in the early stages of processing. Nonetheless, social stimuli demand more cognitive resources in later stages involving cognitive control and response selection (200 ms after stimulus onset). Authors postulate that this is probably the result of interpreting 
social information. Thus, evidence suggests that directional non-social and social signals induce cognitive conflict; however, the nature underlying the differences between them remains unclear.

In another study, Ricciardelli and colleagues (2002) investigated the effect of social signals on an instructed saccade task. Here participants were instructed to program a saccade after a cue to a location while a flanked distractor was presented. The distractor was a face that gazed congruently or incongruently to the instructed saccade direction. Results showed that participants were faster and more accurate when the distractor's gaze was spatially congruent with the landing location (Ricciardelli et al., 2002). These findings have been replicated employing different cue types and distractors (e.g., Ciardo et al., 2014; Dalmaso et al., 2020; Dalmaso, Galfano, \& Castelli, 2015; Porciello, Liuzza, Minio-Paluello, Caprara, \& Aglioti, 2016; Dalmaso et al., 2020a; Kuhn et al., 2010; Hietanen, 2018). Altogether, this collection of studies indicates that gaze stimuli can interfere extensively with oculomotor planning and execution due to the cognitive conflict that they elicit. Despite the robust findings, studies have yet to examine what are the underlying cognitive mechanisms in the presence of conflicting social signals in socially interactive situations.

The present study aimed to understand how the human brain responds to conflicting social signals during a goal-oriented task that required cognitive control on a simulated object handover. To do so, we adapted Admoni and colleagues' (2014) handover task. Participants completed a screen-based categorization task where they interacted with a robot that "handed" them differently colored objects. The objects were either completely (100\%) blue (or yellow), half blue/half yellow (50\% blue/yellow), or predominantly one color ( $75 \%$ blue/25\% yellow or $75 \%$ yellow/25\% blue). After the 
simulated handover, the robot executed a head-cue towards one of two bins (i.e., a yellow bin on the left or a blue bin on the right, which were counterbalanced). The robot's signals were either congruent or incongruent with the objects' color. Participants categorized the object depending on the color of the object, irrespective of the social cue. We measured performance in terms of reaction times, accuracy and the neural correlates of cognitive conflict when observing social signals that conflict with the goal of a task. Since conflict arises due to the incompatibility between the expected and the robot's actual behavior (e.g., looking at the yellow bin when the object is mostly blue), we hypothesized that incongruent social signals' processing would elicit behavioral and neural indices of cognitive conflict. We measured cognitive conflict by examining participants' motor and oculomotor responses (i.e., Error rates and reaction times; Study 1 and Study 2), gaze-path behavior (i.e., reaction times and gaze-path analysis; Study 2), and electrophysiological markers of cognitive conflict (i.e., ERPs and ERSP; Study 3).

\section{Study 1}

Study 1 aimed to examine if participants would indeed exhibit behavioral effects of cognitive conflict in the new paradigm designed to address our theoretical question. If participants do experience conflict when viewing head movements incongruent with expectations and task goals, we would expect longer RTs and more errors compared to when viewing congruent head movements.

\section{Methods}

Participants. We conducted an a priori power analysis for the effect of congruency, using: (i) a moderate effect size (Cohen's $\mathrm{dz}=.6$ ) according to effect sizes found in previous studies (Perez-Osorio et al., 2015; Perez-Osorio et al., 2017), (ii) an $\alpha-$ 
error equal to .05, and (iii) a power level of .95 (as recommended by Cohen, 1992). This analysis yielded an adequate sample size of 37 participants. We recruited 37 participants online using Prolific (Palan \& Schitter, 2018). The median age was 27 years; 34 participants were right-handed; 19 were females, 15 were males. A sensitivity analysis was conducted to examine the critical $\mathrm{F}$ and demonstrated that considering numerator $\mathrm{df}=3, \alpha=0.05$ and $1-\beta=0.95$, the minimum effect size detectable should be $\mathrm{F}=\mathbf{2 . 8 5}$, which is smaller than the observed f statistic; see the results of Study 1. Participants were compensated $£ 1.88$ upon completion. Participants were naïve to the aims of the experiment but were debriefed upon its completion. The experiment was conducted in accordance with the 2013 declaration of Helsinki and was approved by the local ethical committee (Comitato Etico Regione Liguria). Raw data can be found on the OSF page of the study https://osf.io/rpb95/.

Apparatus. The stimuli were created by taking photographs of the humanoid robot iCub (Metta et al., 2010) in various head positions while manipulating the object. $\mathrm{iCub}$ is an open-source humanoid robot platform designed to investigate the development of artificial intelligence. It is $104 \mathrm{~cm}$ in height with 53 degrees of freedom, with articulated head and eyes and dexterous manipulation capability (Metta et al., 2010). The experiment was programmed using Psychopy (Peirce et al., 2019) and uploaded to the Pavlovia repository.

Task. Participants completed an object categorization task where they saw one of 5 objects (i.e., a 100\% blue object, a 75\% blue and 25\% yellow object, a 50\% blue and $50 \%$ yellow object, a 100\% yellow object, and a $75 \%$ yellow and $25 \%$ blue object). After seeing the object, participants decided if it belonged to one of two categories (i.e., the blue category or the yellow category). Each trial started with a fixation presented for 
1000 ms. Next, two differently colored "bins" appeared on the top corners of the screen (i.e., one bin on the top-right and the other on the top-left of the screen) and remained on the screen until the end of the trial. $1000 \mathrm{~ms}$ later, iCub appeared in the middle of the screen, looking straight ahead with one of the objects in front of it. After looking straight for $400 \mathrm{~ms}$, iCub looked down at the object for $1000 \mathrm{~ms}$. Next, an image of iCub reaching for the object was presented for $500 \mathrm{~ms}$, followed by iCub grasping the object for 500 ms. Afterward, an image of iCub extended its arm to indicate handing over the object to the participant was presented. $500 \mathrm{~ms}$ later, iCub looked either at the correct bin (i.e., congruent trial) or at the incorrect bin (i.e., incongruent trial). Participants were instructed to wait until iCub completed the head movement to categorize the object by pressing one of two keys, " $K$ " for the right bin and " $D$ " for the left bin. If participants did not respond within $3000 \mathrm{~ms}$, the trial timed out. After categorizing the object based on color, participants received feedback (i.e., "Correct" or "Incorrect"). The inter-trial interval (ITI) was set to $1000 \mathrm{~ms}$.

The bins' position was counterbalanced across the entire experiment such that the yellow bin was on the top-right corner and the blue bin was on the left-top corner $50 \%$ of the time and vice versa. The $100 \%$ blue object was presented 8 times, the $100 \%$ yellow object was presented 8 times, the $75 \%$ blue object was presented 16 times, the $75 \%$ yellow object was presented 16 times and the 50\% colored object was presented 8 times (i.e., 56 objects). Feedback was always presented as "correct" for the $50 \%$ object.

In addition to the experimental trials, we included 6 catch trials where the robot looked down, and the participants had to respond by pressing the spacebar. The catch trials ensured that participants were paying attention throughout the study. The experiment contained 62 trials overall and was divided into two blocks with a self-paced 
rest between the blocks (i.e., each block contained 31 trials) and took approximately 20 minutes to complete. The order of the trials was pseudorandomized online for all the participants. See Figure 1 for the trial sequence.

The bins' position was counterbalanced across the entire experiment such that the yellow bin was on the top-right corner and the blue bin was on the left-top corner $50 \%$ of the time and vice versa. The $100 \%$ blue object was presented 8 times, the $100 \%$ yellow object was presented 8 times, the $75 \%$ blue object was presented 16 times, the $75 \%$ yellow object was presented 16 times and the 50\% colored object was presented 8 times (i.e., 56 objects). Feedback was always presented as "correct" for the $50 \%$ object.

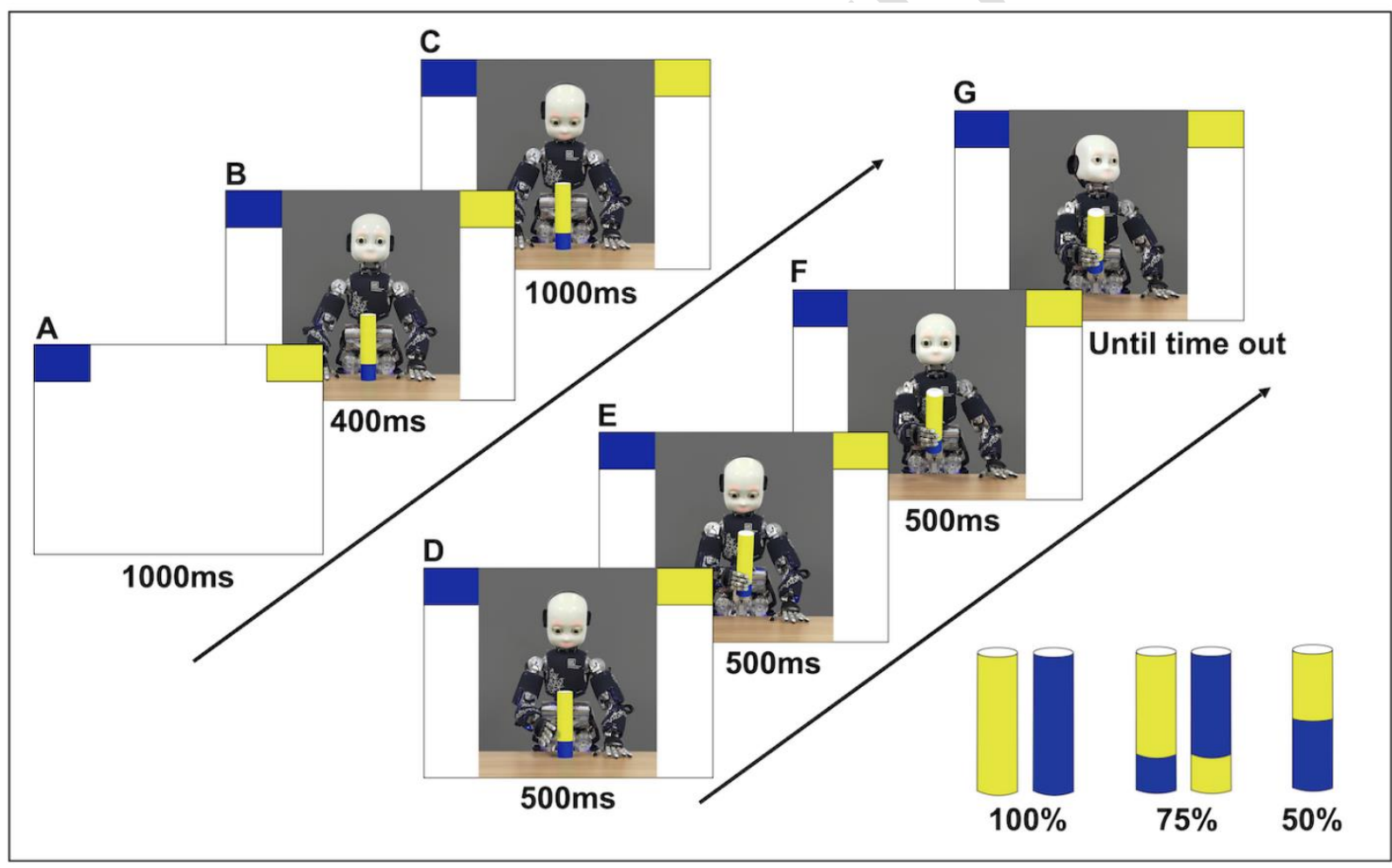

Figure 1. Trial sequence of the categorization task. The figure illustrates the task sequence from left to right. Each trial started with a fixation cross shown for 10ooms (not shown in the figure). Next, the "bins" would appear on the screen, followed by iCub appearing to look straight ahead. iCub would then complete a series of sequences to mimic handing over an object. After extending its' arm to illustrate the handover, iCub would either look congruently at the target location or in the opposite direction. Timeout was set to $3000 \mathrm{~ms}$, and the ITI was 1000ms. The type of objects used in the categorization task is depicted on the bottom right. 
In addition to the experimental trials, we included 6 catch trials where the robot looked down, and the participants had to respond by pressing the spacebar. The catch trials ensured that participants were paying attention throughout the study. The experiment contained 62 trials overall and was divided into two blocks with a self-paced rest between the blocks (i.e., each block contained 31 trials) and took approximately 20 minutes to complete. The order of the trials was pseudorandomized online for all the participants. See Figure 1 for the trial sequence.

Procedure. After providing consent, participants were instructed to categorize objects based on their color as quickly and accurately as possible. They were told that they would see a robot that would hand them an object and look at one of two bins on each trial. After seeing the robot's head move, they were instructed to select the appropriate bin. After completing the practice block, which consisted of five trials, participants moved to the experimental block. After completing the task, participants saw a unique completion code to ensure that they completed the study.

Analysis. To examine the interference effect exerted on participant's motor responses, we examined both reaction times (RTs) and error rates. To calculate average RTs, we first excluded incorrect trials, and then excluded trials shorter than $50 \mathrm{~ms}$ and longer than $1500 \mathrm{~ms}$. Next, we excluded RTs that were beyond 3 SDs of each factorial combination within each participant. Erroneous trials constituted only $10 \%$ of the trials. The remaining $90 \%$ of the data were averaged to calculate mean RTs. We calculated a mean percentage of error for each factorial combination for each participant to calculate error rates. Next, we ran two within-factor 2 x 2 repeated-measures ANOVAs with Congruency (congruent vs. incongruent) and Object type (100\% object vs. $75 \%$ object) as factors (i.e., each ANOVA examined differences in RTs and error rates separately). 
The 50\% object was examined separately using a chi-squared test, as including it in the ANOVA would violate assumptions regarding collinearity. Specifically, we examined if the frequency of compliance with the robot depended on iCub's head signals. In other words, we tested if rightward signals were associated with more right-bin choices and vice-versa.

\section{Results}

One participant was excluded from data analysis due to incorrect responses for all $75 \%$ objects. Data for the remaining 36 participants were analyzed. The ANOVA examining differences in average RTs showed a significant effect of Congruency $(F(1,35)$ $\left.=25.74, \eta^{2} \mathrm{G}=.02, p<.001\right)$ with faster responses for congruent vs. incongruent trials, but no effect of Object type $\left(F(1,35)=2.88, \eta^{2} \mathrm{G}=.001, p=.09\right)$, or Congruency $\mathrm{X}$ Object type interaction $\left(F(1,35)=.19, \eta^{2} \mathrm{G}<.01, p=.66\right)$. The ANOVA examining differences in error rates revealed a significant effect of Congruency $(F(1,35)=15.26$, $\eta^{2} \mathrm{G}=.11, p<.001$ ) with higher error rates for incongruent trials vs. congruent trials. The ANOVA also revealed a significant effect of Object type $\left(F(1,35)=10.41, \eta^{2}{ }_{\mathrm{G}}=.01, p\right.$ $<.01$ ), with higher error rates for the $75 \%$ objects compared to the $100 \%$ objects. The Congruency X Object type interaction was also significant $\left(F(1,35)=6.23, \eta^{2} \mathrm{G}<.001, p\right.$ $=.01)$ such that differences in the proportion of error rates between congruent and incongruent trials were larger for the $75 \%$ object compared to the $100 \%$ object; see Table 1 and Figure 2 for the mean RTs and error rates. 
Manuscript accepted for publication in the Journal of Cognitive Neuroscience - JoCN

doi: 10.1162/jocn_a_o1786

\section{Table 1.}

Means of error rates and RTs from Study 1.

\begin{tabular}{lllllllll}
\hline & \multicolumn{7}{l}{$100 \%$ Object } & \multicolumn{2}{l}{$75 \%$ Object } \\
\hline & \multicolumn{2}{l}{ Congruent } & \multicolumn{2}{l}{ Incongruent } & \multicolumn{2}{l}{ Congruent } & \multicolumn{2}{l}{ Incongruent } \\
\cline { 2 - 9 } & M & SD & M & SD & M & SD & M & SD \\
Error rate & $3.25 \%$ & 7.58 & $11.3 \%$ & 16.5 & $5.19 \%$ & 7.3 & $16.7 \%$ & 19.3 \\
RT & 461 & 278 & 534 & 308 & 482 & 303 & 544 & 351 \\
\hline
\end{tabular}

Note. The table shows the mean error rates and RTs of the $2 \times 2$ ANOVAs. Reaction time units are in milliseconds (ms).
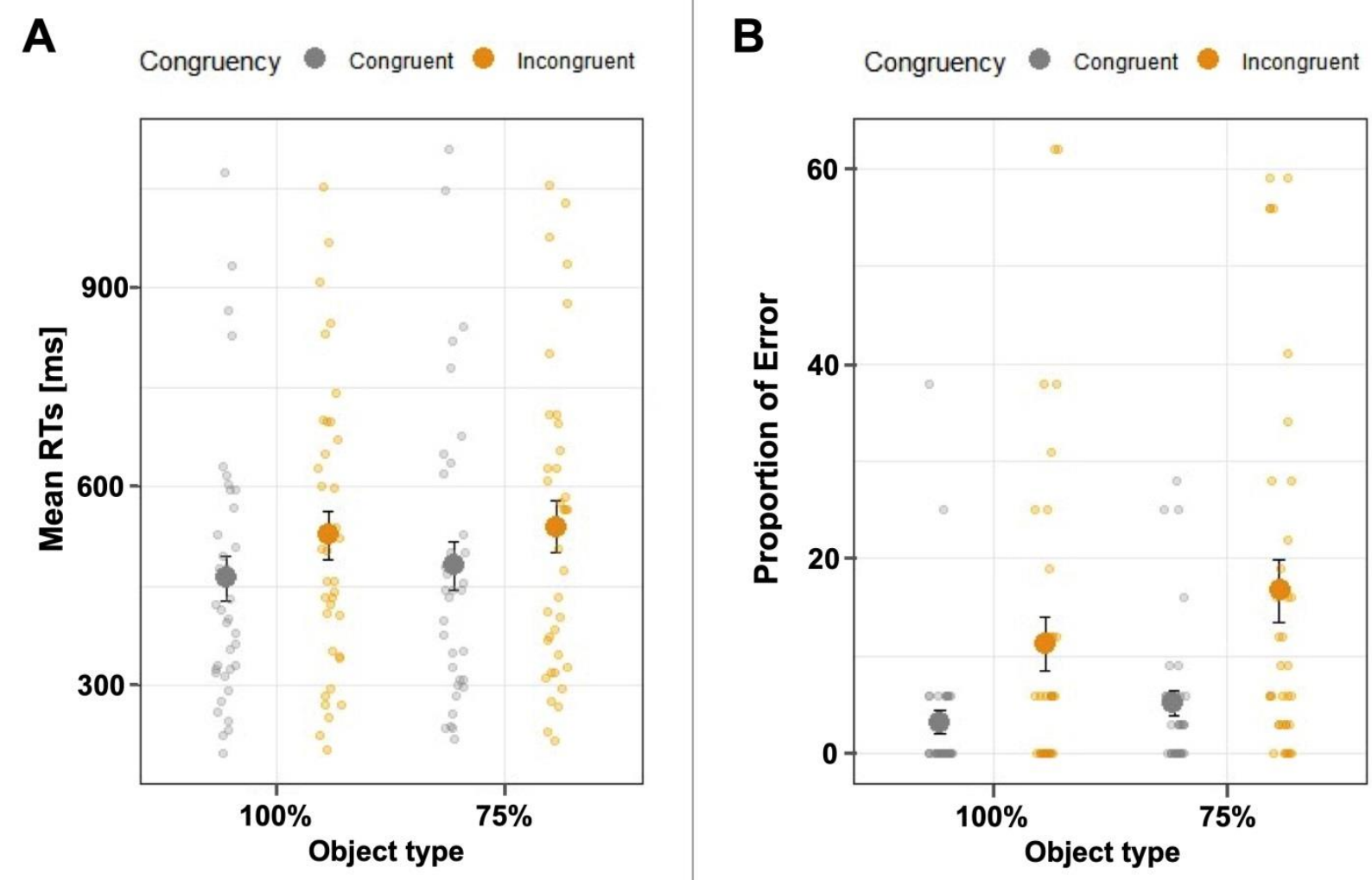

Figure 2. Reaction Times and Error Rates as a function of congruency and object type. The graph illustrates raw and averaged data in the 2 x 2 ANOVA. The graphs show mean RTs of responses (left panel) and the proportion of error (right panel) as a function of congruency and object type. Analysis of RTs shows faster RTs for congruent vs. incongruent signals. Analysis of error rates shows larger differences between congruent and incongruent signals for the $75 \%$ object compared to the $100 \%$ object. Error bars illustrate the Standard Error of the Mean. 


\section{Discussion Study 1}

The purpose of Study 1 was to examine the impact of incongruent gaze behavior on behavioral responses. Participants completed a simulated handover task with a robot and were instructed to categorize objects based on their color. After the robot handed over the object, it looked congruently at the correct bin (i.e., the object was predominantly yellow and the robot looked at the yellow bin) or incongruently at the incorrect bin (i.e., the object was predominantly yellow and the robot looked at the blue bin). The results showed that participants were slower at categorizing the object when the robot looked at the incorrect bin compared to when it looked at the correct bin. The results also showed that participants made more errors in identifying which bin was appropriate when the robot looked at the incorrect bin relative to when it looked at the correct bin. This suggests an interference effect in participants' motor responses, which provides evidence for cognitive conflict. The error rates' effect was more pronounced for the more ambiguous objects ( $75 \%$ color), relative to those that were of one color only (100\% blue or yellow). This is not surprising, as participants probably followed iCub's head shift when deciding where to place the most ambiguous objects ( $75 \%$ and $50 \%$ ).

\section{Study 2}

Study 2 aimed at replicating the effect of cognitive conflict observed in Experiment 1 with a different dependent variable, namely eye movement patterns. This should allow not only for replication of the effect we observed in Study 1, but also for examining if the cognitive conflict induced in our paradigm affects the oculomotor system. Since online studies come with the limitation of not collecting eye movement data reliably, Experiment 2 aimed to examine saccadic RTs, saccadic error rates, and gaze-path trajectories as indices of cognitive conflict in the laboratory. We examined 
differences in saccadic reaction times (RT) and error rates (i.e., fixations on the wrong location) to replicate the manual response time findings of Study 1. As such, we expected to find faster saccadic RTs for congruent vs. incongruent signals, more errors for incongruent vs. congruent signals with large congruency differences for the $75 \%$ object compared to the $100 \%$ object. For gaze-path trajectories, we expected that if the experienced conflict also affects the oculomotor system, observers would treat the head cue as a distractor from completing the task, and as such, we would expect differences in gaze-path trajectories when viewing incongruent vs. congruent head cues. We did not have a directional hypothesis regarding gaze-path trajectories, as theories predict different curvature patterns for distractor objects depending on the paradigm (e.g., if one distractor is present, the gaze path deviates towards an object but deviates away if two distractors are present). Therefore, we were unable to make a directional hypothesis regarding gaze-path trajectories (see Van der Stigchel et al., 2006 for a review).

\section{Methods}

Participants. We conducted an a priori power analysis, using: (i) the effect size of the main congruency effect from Study 1 (Cohen's d =1.08), (ii) an $\alpha$-error equal to .05 , and (iii) a power level of .95. The analysis yielded a sample size of 14 participants. However, in Study 2 we changed the response type from key presses to eye movements. To compensate for possible differences due to the change in the response type, we opted for a sample size of 20 participants. Twenty volunteers were recruited (Median age $=$ 30; 28 right-handed; 13 females, 15 males). A sensitivity analysis was conducted to examine the critical $\mathrm{F}$ and demonstrated that considering numerator $\mathrm{df}=3, \alpha=0.05$ and 1- $\beta=0.95$, the minimum effect size detectable should be $F=3.09$. All participants had a normal or corrected vision (contact lenses) and signed informed consent before 
starting the experiment. Participants were naïve to the aims of the experiment but were debriefed upon completion of the experiment. The experiment was conducted in the S4HRI lab of Istituto Italiano di Tecnologia (Genova, Italy) in accordance with the 2013 declaration of Helsinki and was approved by the local ethical committee (Comitato Etico Regione Liguria).

Apparatus and eye-tracking acquisition. The experiment was designed and presented using OpenSesame (Mathôt et al., 2012). Participants were seated $65 \mathrm{~cm}$ away from a 19-inch monitor $(1920 \times 1200 \mathrm{px}, 60 \mathrm{~Hz})$. During the experiment, an eyetracker (Eyelink 1000, SR Research, Ontario, CA) recorded the right eye position (spatial resolution of $0.1^{\circ}, 1000 \mathrm{~Hz}$ ). A 9-point standard calibration was conducted before each block. The block started only when the difference between the calibration point and the fixation was less than $1^{\circ}$ on average. To collect the responses, we defined 3 circular AOIs on the face and the bins (radius $=100 \mathrm{px}$ ), and a vertical ellipse AOI on the object (width $=170 \mathrm{px}$; height $=300 \mathrm{px}$ ). Data was exported using Eyelink Data Viewer (SR Research, Ontario, CA).

Task and Procedure. Participants performed the same task as in Study 1 except that they responded by looking at the correct bin instead of pressing the button corresponding to the correct bin. Each trial started with a fixation presented for $500 \mathrm{~ms}$. Next, the bins appeared on the top corners of the screen and remained on the screen until the end of the trial. $500 \mathrm{~ms}$ later, iCub appeared in the middle of the screen, looking straight ahead for a random time between 800 and $1200 \mathrm{~ms}$. Then iCub looked down at the object for $500 \mathrm{~ms}$. Next, a sequence of images of the robot reaching, grasping, and handling the object were presented, each one for $500 \mathrm{~ms}$ (cf. Figure 1). Later, participants were instructed to fixate at iCub's face; only then the robot looked 
either at the correct bin (i.e., congruent trial) or at the incorrect bin (i.e., incongruent trial). After iCub's head shift, participants had to respond with a saccade to the correct bin. If participants did not respond within $2000 \mathrm{~ms}$, the trial timed out. After categorizing the object based on color, participants received feedback (i.e., "Correct", "Incorrect", or "Please respond faster") for $500 \mathrm{~ms}$.

Similar to Study 1, the bins' position was counterbalanced across the entire experiment such that the yellow bin was on the top-right corner and the blue bin was on the left-top corner $50 \%$ of the time and vice versa. The $100 \%$ blue object was presented 16 times, the $100 \%$ yellow object was presented 16 times, the $75 \%$ blue object was presented 32 times, the $75 \%$ yellow object was presented 32 times and the $50 \%$ colored object was presented 16 times (i.e., 112 trials in total). Feedback was always presented as correct for the 50\% object. In Study 2, we also included 12 catch trials, where the robot looked down to ensure that participants were paying attention throughout the experiment. Participants had to respond by fixating on the object; any other response was considered an error. The study consisted of two blocks of 62 trials, each with a selfpaced rest between the blocks, for a total of 124 trials. All the participants performed a practice block of 12 trials before the actual experiment. In total, the experiment took approximately 25 minutes to complete, including the calibration. The trial order was pseudorandomized before the experiment and divided into blocks with an equal number of trials.

Analysis. To analyze saccadic RTs, we first excluded incorrect trials (i.e., fixations landing on the wrong location), with saccades faster than $50 \mathrm{~ms}$ and longer than $1500 \mathrm{~ms}$. We then removed saccades beyond 3 SDs within each factorial combination within each participant and finally averaged the duration of the saccades 
for all the trials. Erroneous trials constituted $17 \%$ of the data and were removed from the analyses. We examined differences in saccadic RT using a within-factor 2 x 2 repeatedmeasures ANOVAs with Congruency (congruent vs. incongruent) and Object type (100\% object vs. $75 \%$ object) as factors. To analyze saccadic error rates, we removed saccades that were faster than $50 \mathrm{~ms}$ and longer than $1500 \mathrm{~ms}$, as trials outside this range could result from technical issues regarding the eye-tracker. We then calculated the proportion of saccadic error for each participant/condition combination. Next, we planned to examine differences in saccadic error rates using a within-factor $2 \times 2$ repeated-measures ANOVAs with Congruency (congruent vs. incongruent) and Object type (100\% object vs. $75 \%$ object) as factors. To examine differences in participant's gaze-path trajectories, the data was down sampled to $100 \mathrm{~Hz}$ in preprocessing. Then, we normalized the saccadic samples of the $\mathrm{x}$-horizontal axis to -1 to 1 . Finally, we calculated the quadratic curvature of participant's gaze behavior, with larger absolute quadratic coefficients illustrating larger curvature in gaze-path trajectories (Tudge et al., 2017 and Ludwig \& Gilchrist, 2002). To do so, we first normalized the $\mathrm{X}$-axis coordinates to collapse over trajectories that ended on the left side and the right side for all correct trials. Next, we fit a quadratic function for each factor-combination within each participant. Then, we extracted the estimate of the quadratic term estimates and examined if the estimates were significantly different between the conditions (i.e., Congruency and Object type). Quadratic coefficients are interpreted such that the absolute value of the quadratic coefficient indicates how large or small the curvature is. Specifically, larger coefficients indicate larger curvatures and vice versa. Finally, we ran a linear mixed model predicting the quadratic coefficient from Congruency (congruent 
vs. incongruent) and Object type (100\% vs. $75 \%)^{1}$ as two dummy coded variables and their interaction. The model contained a random intercept for each participant. We ran a second analysis to examine differences in saccadic RTs.

\section{Results}

Analysis of the saccadic RTs showed a significant effect of Congruency $(F(1,19)=$ $12.28, \eta^{2} \mathrm{G}=.01, p=.002$ ), with faster saccadic RTs for congruent vs. incongruent trials. However the effect of Object type $\left(F(1,19)=.02, \eta^{2} \mathrm{G}<.001, p=.89\right)$, and the Congruency X Object type interaction were not significant $\left(F(1,19)=.14, \eta^{2} \mathrm{G}<.001, p=\right.$ .71); see Table 2 and Figure 3. While the analysis plan for saccadic error rates followed Study 1, we were unable to run inferential statistics as the congruent/100\% object condition had zero variance. As such, we simply descriptively report means and standard deviations.

The congruent $/ 100 \%$ object had the lowest error rate $(\mathrm{M}=0, \mathrm{SD}=0)$, the congruent $/ 75 \%$ object had an error rate of $\mathrm{M}=.48 \%, \mathrm{SD}=.01$, the incongruent $/ 100$ object had an error rate of $.97 \%, \mathrm{SD}=.02$ and the incongruent $/ 75 \%$ object had a mean error rate of $2.2 \%, \mathrm{SD}=.02$. While information regarding statistical significance cannot be determined in this case, the pattern of results seems to be in line with the results of manual error rates of Experiment 1: there does seem to be a larger descriptive difference in error rates between congruent and incongruent head movements, and this difference appears to be larger for the $75 \%$ object (i.e., error rate difference $=1.72 \%$ ) vs. the $100 \%$ object (i.e., error rate difference $=.97 \%$ ).

\footnotetext{
${ }^{1}$ We ran a linear mixed model because we did not have equal number of observations for each cell, which violates ANOVA assumptions.
} 
Manuscript accepted for publication in the Journal of Cognitive Neuroscience - JoCN

doi: 10.1162/jocn_a_o1786

Table 2.

Quadratic coefficients, saccadic RTs and saccadic error rates of Experiment 2.

\begin{tabular}{|c|c|c|c|c|c|c|c|c|}
\hline & \multicolumn{4}{|c|}{$100 \%$ Object } & \multicolumn{4}{|c|}{ 75\% Object } \\
\hline & \multicolumn{2}{|c|}{ Congruent } & \multicolumn{2}{|c|}{ Incongruent } & \multicolumn{2}{|c|}{ Congruent } & \multicolumn{2}{|c|}{ Incongruent } \\
\hline & M & $\mathrm{SD}$ & M & $\mathrm{SD}$ & M & $\mathrm{SD}$ & M & $\mathrm{SD}$ \\
\hline $\begin{array}{l}\text { Quadratic } \\
\text { coefficient }\end{array}$ & $-2.11^{-4}$ & $7 \cdot 99^{-5}$ & $-2.48^{-4}$ & $7 \cdot 61^{-5}$ & $-2.25^{-4}$ & $7 \cdot 99^{-5}$ & $-2.27^{-4}$ & $8.83^{-5}$ \\
\hline Saccadic RT & 390 & 136 & 425 & 165 & 393 & 147 & 422 & 166 \\
\hline $\begin{array}{l}\text { Saccadic } \\
\text { error rate }\end{array}$ & O\% & $\mathrm{O}$ & $.97 \%$ & .02 & $.48 \%$ & .01 & $2.20 \%$ & .02 \\
\hline
\end{tabular}

Note. Saccadic RTs are in milliseconds
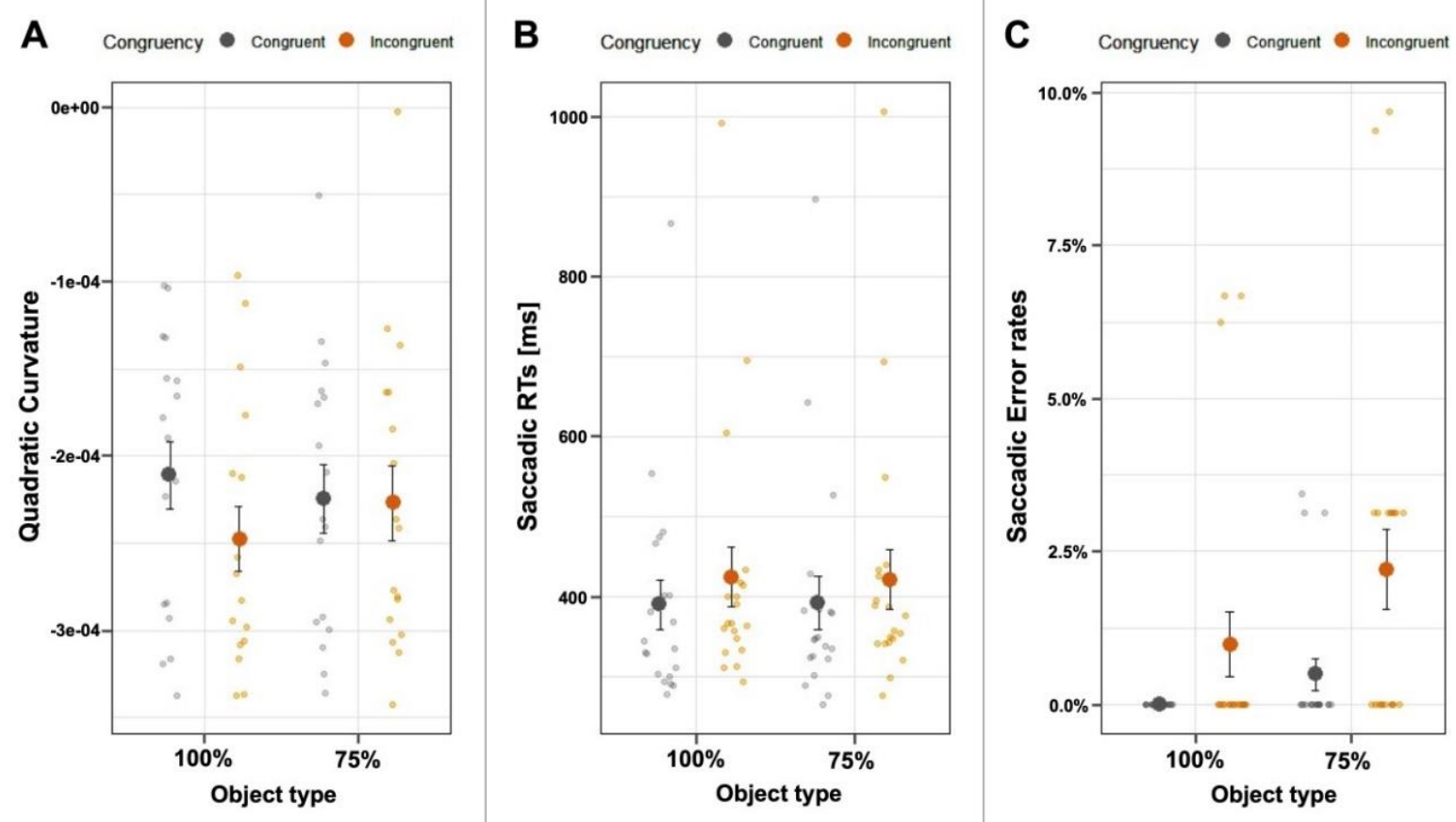

Figure 3. Quadratic coefficients of the gaze trajectories: Panel A illustrates the differences in quadratic coefficients of quadratic functions based on the congruency of iCub head movement for the 100\% object and the $75 \%$ object. Panel B shows the saccadic reaction times for congruent/incongruent head movements for each of the $100 \%$ and $75 \%$ objects. Finally, panel C shows saccadic error rates. The error bars illustrate the standard error of the mean.

Analysis of the quadratic coefficient using the linear mixed model showed a significant intercept $\left(b=-2.11^{-4}, \mathrm{SE}=1.96^{-5}, t(20.81)=-10.71, p<.001\right)$, a significant 
effect of Congruency $\left(b=-3.65^{-5}, \mathrm{SE}=1.14^{-5}, t(48)=-3.19, p=.002\right)$ with larger quadratic estimates for congruent vs. incongruent trials, but no significant effect of Object type $\left(b=-1.35^{-5}, \mathrm{SE}=1.14^{-5}, t(48)=-1.18, p=.24\right)$. However, the model showed also a significant Congruency X Object type interaction $\left(b=3.43^{-5}, \mathrm{SE}=1.61^{-5}, t(48)=\right.$ $2.12, p=.03)$. To see if the gaze path curved away or towards the robot's face, we plotted the mean normalized absolute value of the $\mathrm{X}$ - and $\mathrm{Y}$-axis for each of the condition. From observing the plots, it seems that both conditions curved away from iCub's face; see

\section{Figure 4.}

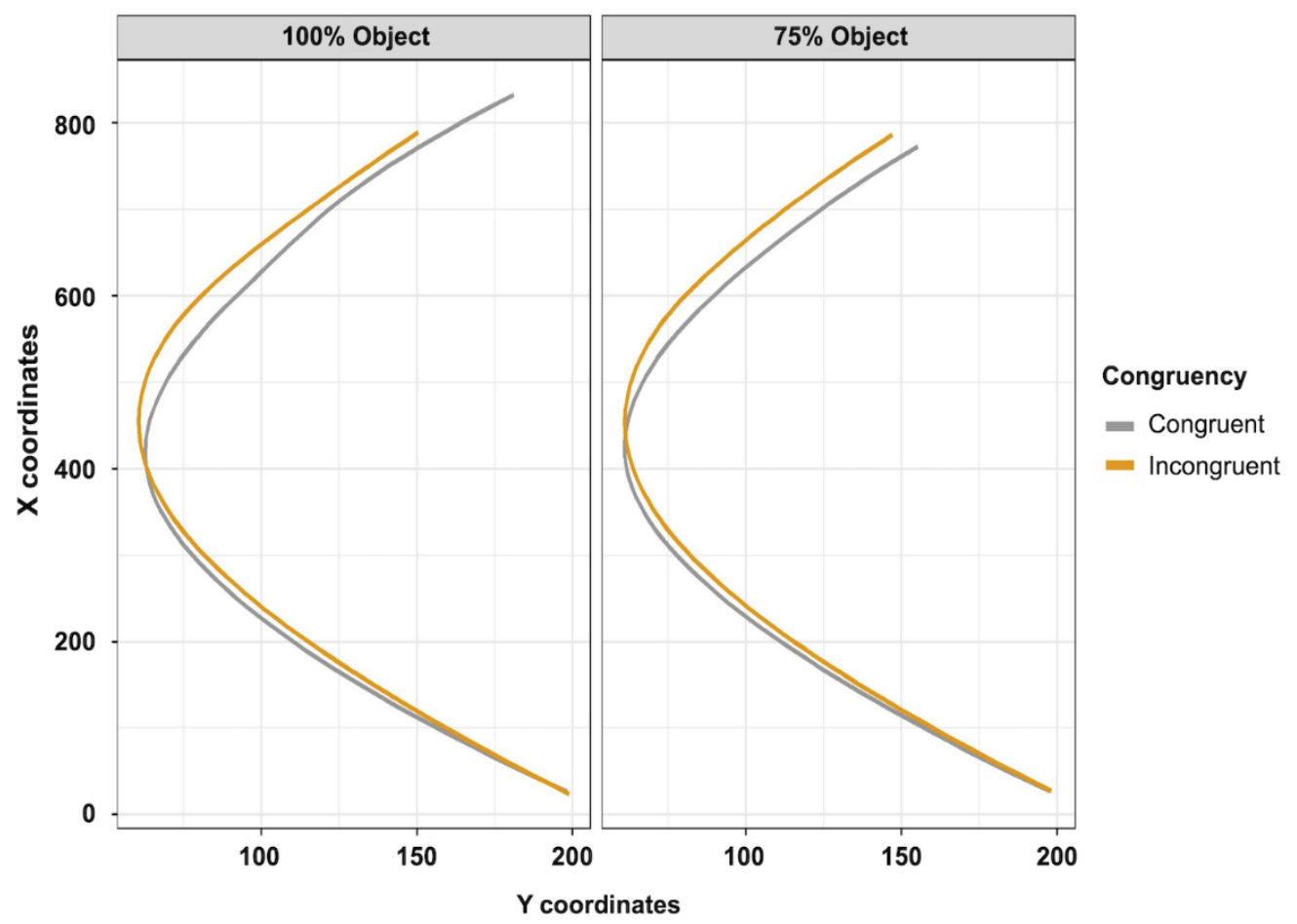

Figure 4. Curve-trajectories of the gaze-path analysis: All conditions show that participants curved away from the robot's face, located in the 200 mark of the y-coordinate.

\section{Discussion}

Study 2 followed similar procedures as Study 1 to examine the effect of incongruent gaze behavior of the depicted iCub robot on participants' behavior, thereby 
addressing cognitive conflict induced by irrelevant social signals. In Study 2 we aimed at corroborating results from Experiment 1 with a different measure, namely eye movement data, instead of manual responses. This would also allow us to examine whether cognitive conflict induced by our manipulation affects the oculomotor system. In Study 2, participants were instructed to make eye movements to where the correct bin was presented. Behavioral results of the eye-tracking experiment (saccadic RTs) replicate the congruency finding from Study 1, showing that responses to congruent head movements were faster than those to incongruent head movements.

Additionally, the experiment results showed that participants had larger curvature in gaze-trajectories when the robot looked incongruently at the target bin compared to when it looked congruently in general. This difference was even more prominent when the object was unambiguous (i.e., larger congruent vs. incongruent curvature differences for the $100 \%$ object vs. the $75 \%$ object). This finding suggests that participants could have curved their gaze towards the robots' signaled direction (and thus away from the correct bin) when it looked incongruently at a target location. This further supports the notion that cognitive conflict is experienced when participants view an incongruently looking robot, and interestingly, cognitive conflict induced by our manipulation affects attentional processing in the oculomotor system.

\section{Study 3}

Study 3 aimed at examining EEG markers of the effect observed in Study 1 and Study 2, following the logic of investigating cognitive conflict induced by irrelevant social signals, with various dependent measures. Since the design of Study 2 was not optimal for electrophysiological data (i.e., too many eye movements could interfere with the EEG signal), we conducted Study 3 tailored to recording EEG data. Specifically, we 
hypothesized that when viewing incongruent head movements, more negative N2 amplitudes would be observed in the event-related potentials (ERP) of the EEG signal and more frontal theta in event-related spectral perturbations (ERSP).

\section{Methods}

Participants. We conducted an a priori power analysis using: (i) the large effect size of the main congruency effect from Study 2 (Cohen's $\mathrm{dz}=.92$ ), (ii) a high $\alpha$-error equal to .001 to prevent the low power typically found on EEG/ERPs studies (Clayson et al., 2019), and (iii) a power level of .95. The analysis yielded a sample size of 32 participants. Anticipating possible exclusions due to the excess of artifacts, we recruited a total of thirty-four participants for Study 3 (Median age = 23; all right-handed; 27 females, 7 males). A sensitivity analysis was conducted to examine the critical $\mathrm{F}$ and demonstrated that considering numerator $\mathrm{df}=3, \alpha=0.05$ and $1-\beta=0.95$, the minimum effect size detectable should be $F=2.88$. All the participants received compensation of $€ 35$ upon completion, were naive to the experiment's aims and were debriefed upon completion of the experiment. The experiment was conducted in the S4HRI lab of Istituto Italiano di Tecnologia (Genova, Italy), in accordance with the 2013 declaration of Helsinki and was approved by the local ethical committee (Comitato Etico Regione Liguria).

Apparatus. The handover task was programmed using OpenSesame (Mathot et al., 2012) and presented on a 27-inch screen with a refresh rate of $80 \mathrm{~Hz}$. Responses were recorded and collected using a standard keyboard.

Task and Procedure. The experiment followed the same sequence as Study 1 and 2 with only changes in the number of trials. Specifically, participants completed 96 trials of $100 \%$ objects (i.e., 48 trials for the blue object and 48 trials for the yellow 
object), 96 trials for the $75 \%$ blue object and 96 trials for the $75 \%$ yellow object, and 96 trials for the $50 \%$ object. The robot looked in the same direction as the correct bin $50 \%$ of the time (i.e., 192 trials) and 50\% of the time in the correct bin's opposite direction (i.e., 192 trials). The experiment also had trials where the robot looked down, which constituted catch trials (i.e., 36 trials). The experiment had 420 trials overall and took about 35 minutes to complete. Previous studies that measured anterior N2 ERPs and conflict-related ERSPs (Cavanagh et al., 2012; Cohen \& Donner, 2013; Botvinick et al., 2001) used a minimum of 100 trials per condition. However, we wanted to compare the results of Study 1 and 2 with the EEG study. Thus, we opted for 96 trials per object (100\% blue/yellow, $75 \%$ yellow, $75 \%$ blue, and 50/50 yellow/blue) and catch trials $(\sim 10 \%)$. We estimated an average of $10-20 \%$ trial exclusion due to poor performance and artifacts. The order of the trials was pseudorandomized before the experiment and divided into blocks with equal number of trials.

Once participants provided their consent, participants were fitted with an EEG cap (Brain Products, $\mathrm{GmbH}$ ) and were given instructions on how to complete the experiment. After doing so, participants completed a practice block, which was succeeded by the experimental part of the study. After completing the experiment, participants were thanked and debriefed.

EEG acquisition and preprocessing. EEG data were recorded using the AgAg-Cl electrodes from a 64 channel electrode system (ActiCap, Brain Products GmbH, Munich, Germany). The data were referenced online to the $\mathrm{Cz}$ electrode location. Ocular movements were recorded using active electrodes located on the F9 and F10 positions for lateral movements and Fp1 and Fp2 for vertical movements. The data were recorded 
and amplified using a BrainAmp amplifier at a sampling rate of $500 \mathrm{~Hz}$ with impedances below $10 \mathrm{k} \Omega$. No filters were applied online during the recording.

EEG preprocessing was conducted using BrainVision Analyzer. As a first step, the EEG signal was down-sampled to $250 \mathrm{~Hz}$, re-referenced to the average of the two mastoids, and then a band filter of .1 $\mathrm{Hz}$ to $30 \mathrm{~Hz}$ was applied. Next, we created epochs that were locked to the onset of the robot's head shift. The epochs started $1000 \mathrm{~ms}$ prior to the head shift and ended $7500 \mathrm{~ms}$ after the shift (i.e., epochs were $8500 \mathrm{~ms}$ long). An Independent Components Analysis (ICA) was conducted on the epochs of interest to isolate and reject components related to blinks and saccades (1-2 components were rejected for each participant at most). Finally, we rejected artifacts with a maximal voltage of $20 \mu \mathrm{V} / \mathrm{ms}$, a $200 \mu \mathrm{V}$ difference in value, or a low activity of .5 $\mu \mathrm{V}$ for $100 \mathrm{~ms}$. For the event-related potential (ERP) analysis, the epochs were then shortened to 200 $\mathrm{ms}$ prior to the stimulus and $400 \mathrm{~ms}$ after, baseline corrected, then averaged for each respective condition combination. As for the Event-Related Spectral Perturbation (ERSP) analysis, data were decomposed using Morlet complex waveforms between $1 \mathrm{~Hz}$ and $30 \mathrm{~Hz}$, with 30 logarithmic frequency steps. The data were then re-epoched to start $1000 \mathrm{~ms}$ prior to the stimulus and $2000 \mathrm{~ms}$ after the stimulus and then averaged. Artifact rejection criteria were identical to the ERP analysis. For both ERPs and ERSPs, we rejected $6 \%$ of the trials for the Congruent/100\% object, $5.6 \%$ for Congruent $/ 75 \%$ object, $7 \%$ for the Incongruent $/ 100 \%$ object and $7.3 \%$ for the Incongruent $/ 75 \%$ object.

Behavioral Analysis. Behavioral analyses of the EEG experiment followed the criteria of Experiment 1. Only 3\% of the trials were removed before the analyses. Two repeated-measures within-factor $2 \times 2$ ANOVAs with Congruency (congruent vs. incongruent) and Object type (100\% color vs. $75 \%$ color) as factors were conducted. The 
first ANOVA examined differences in participants' error rates, while the second examined differences in RTs. The 50\% object was examined separately in a chi-squared test as including it in the ANOVA would violate assumptions regarding collinearity.

EEG Analysis. Two repeated within-factor 2 x 2 ANOVA with Congruency (congruent vs. incongruent) and Object type (100\% color vs. $75 \%$ color) as factors were conducted to examine EEG indices of cognitive control. The first ANOVA examined differences in ERP data, which were based on mean amplitudes exported using a $100 \mathrm{~ms}$ window at $\mathrm{FCz}$ (210 to $310 \mathrm{~ms}$ ) around where the N2 peaked (26o ms). The peak was determined by finding the minimum voltage point of the grand average for all conditions between 200 and $300 \mathrm{~ms}$ (minimum voltage, as the N2 has a negative deflection). The second ANOVA explained differences in averaged ERSP data. Averaged ERSPs were exported by finding the maximal voltage point for the grand average of all conditions for the entire duration of the epoch in which the power spectrum peaked for the average frequencies of interest (i.e., 4-7 Hz) and using a $100 \mathrm{~ms}$ window around where theta oscillations peaked (i.e., $248 \mathrm{~ms}$ from: 198 to $298 \mathrm{~ms}$ ).

\section{Results}

Behavioral results. Analysis of RT data showed a significant effect of Congruency $\left(F(1,28)=119.59, \eta^{2} \mathrm{G}=.11, p<.001\right)$, with faster RTs for congruent $(\mathrm{M}=$ $372 \mathrm{~ms}, \mathrm{SD}=75.4)$ vs. incongruent trials $(\mathrm{M}=432, \mathrm{SD}=91.8)$. The analysis, however, showed no significant effect of Object type $\left(F(1,28)=1.15, \eta^{2} \mathrm{G}<.001, p=.29\right)$ or a Congruency $X$ Object type interaction $\left(F(1,28)=.89, \eta^{2}{ } \mathrm{G}<.001, p=.35\right)$. Prior to examining error rates, three outlying participants were removed from error-rate 
analysis (z-score larger than 4) ${ }^{2}$; they were not removed from other analyses. Analysis of error rates on the remaining participants showed a significant main effect of congruency $\left(F(1,28)=12.82, \eta^{2} \mathrm{G}=.15, p=.001\right)$, object type $\left(F(1,28)=14.56, \eta^{2} \mathrm{G}=.06, p<.001\right)$ and an interaction between them $\left(F(1,28)=5.54, \eta^{2} \mathrm{G}=.02, \mathrm{p}=.02\right)$. The interaction suggests larger differences between congruent and incongruent signals for the $75 \%$ object compared to the 100\% object, see Table 3 .

Similar to Experiment 1, the chi-squared test examining the $50 \%$ object showed that the relationship between the robot's head cue location and participant's choice was significant $\left(X^{2}(1, N=30)=115.79, p<.001\right)$, suggesting that participants indeed followed the (irrelevant) robot's head movements.

ERP Results. Results of the 2 x 2 ANOVA examining N2 amplitudes at FCz showed a significant main effect of Congruency $\left(F(1,33)=59.41, \eta^{2} \mathrm{G}=.1, p<.001\right)$ with smaller amplitudes (i.e., less positive) for incongruent trials vs. congruent trials; see Table 3 for mean amplitudes. The effect of Object type was not significant $(F(1,33)=$ 2.26, $\eta^{2} \mathrm{G}=.004, p=.06$ ). More importantly a significant Congruency X Object type interaction $\left(F(1,33)=5.83, \eta^{2} \mathrm{G}=.005, p=.02\right)$ with larger differences between congruent and incongruent trials for the $100 \%$ object compared to the $75 \%$; see Table 3 and Figure 5 .

\footnotetext{
${ }^{2}$ We re-ran the analysis without excluding the 3 participants. The analysis revealed a significant effect of Congruency $(F(1,31)=11.39, p=.001)$, and a non-significant effect of Object type $(F(1,31)=2.01, p=.16)$ or their interaction $(F(1,31)=3.02, p=.09)$. However, due to violations of normality, the standard errors and $p$ values of this analysis should be interpreted with caution.
} 
Manuscript accepted for publication in the Journal of Cognitive Neuroscience - JoCN

doi: 10.1162/jocn_a_o1786

Table 3.

Amplitudes, frequency power, reactions times and error rates of Study 3.

\begin{tabular}{lllllllll}
\hline & \multicolumn{7}{l}{$100 \%$ Object } & \multicolumn{7}{l}{$75 \%$ Object } \\
\hline & \multicolumn{2}{l}{ Congruent } & \multicolumn{2}{l}{ Incongruent } & \multicolumn{2}{l}{ Congruent } & \multicolumn{2}{l}{ Incongruent } \\
\cline { 2 - 10 } & M & SD & M & SD & M & SD & M & SD \\
N2 & 9.01 & 4.81 & 5.74 & 4.24 & 7.95 & 3.80 & 5.81 & 3.53 \\
Mid-trontal theta & .78 & .40 & 1.37 & .81 & .67 & .47 & 1.36 & .72 \\
RT & 376 & 71.3 & 433 & 90.4 & 369 & 80.3 & 431 & 94.5 \\
Error rate & $.37 \%$ & 1.29 & $.81 \%$ & 1.42 & $.37 \%$ & .79 & $1.28 \%$ & 1.49 \\
\hline
\end{tabular}

Note. RTs are in milliseconds. Please note that although the N2 amplitudes are in the positive range, it is a negative-going deflection, with the incongruent condition eliciting a "more negative" deflection.

A

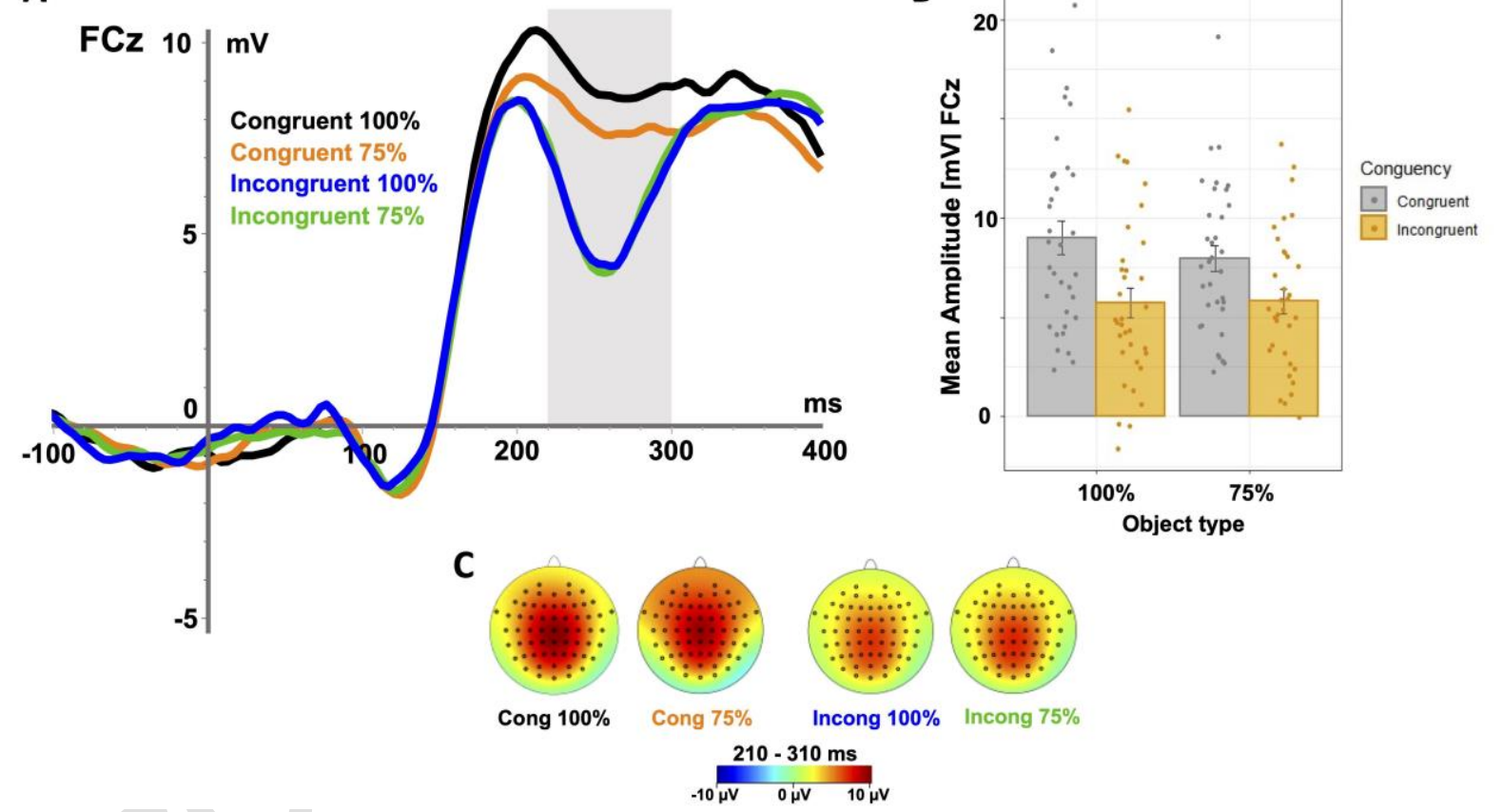

Figure 5. ERP amplitudes as time-locked to the onset of the head cue: The graph illustrates ERP amplitudes at $\mathrm{FCz}$ that were time-locked to the robot's head cue (A). Analysis of the ERP amplitudes showed a significant interaction between congruency and object type where the difference between the congruent and incongruent objects was larger for the $100 \%$ object than the $75 \%$ object (B). The graph also illustrates the topographies for each condition (C).

ERSP Results. Analyses of the ERSPs showed a significant effect of Congruency $\left(F(1,33)=48.92, \eta^{2} \mathrm{G}=.20, p<.001\right)$, with higher ERSP amplitudes in the theta frequency band for incongruent vs. congruent head movements (1.36 $\mu \mathrm{V}$ vs. .73 $\mu \mathrm{V})$, see 
Table 3. The analysis revealed no significant effect of Object type $\left(F(1,33)=1.51, \eta^{2} \mathrm{G}=\right.$ $.002, p=.22)$ or a Congruency X Object type interaction $\left(F(1,33)=.76, \eta^{2}{ }_{\mathrm{G}}<.002, p=\right.$ .39); see Figure 6.

A $\quad \mathrm{FCz}$

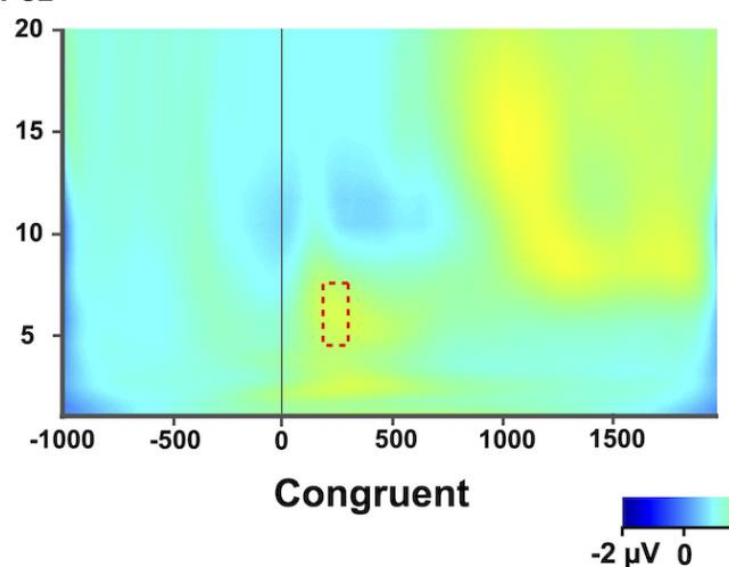

B

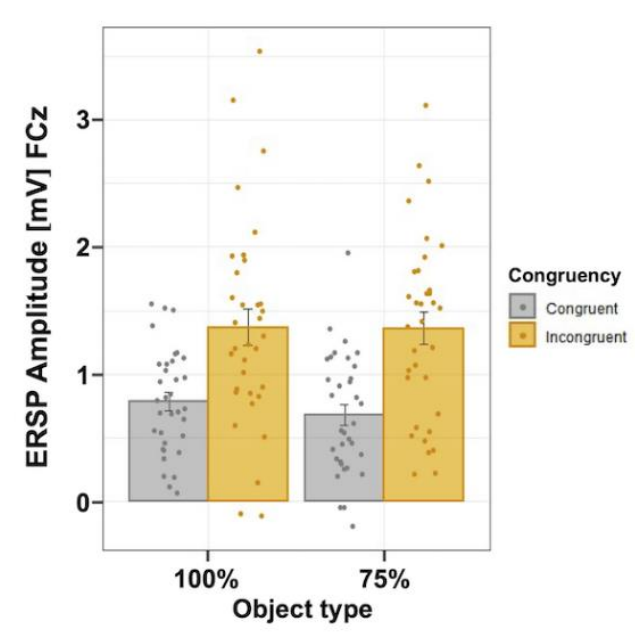

FCz

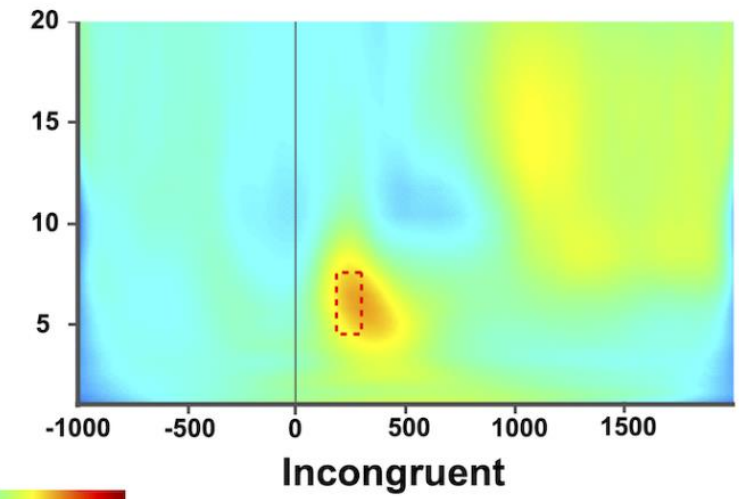

$3 \mu \mathrm{V}$

C

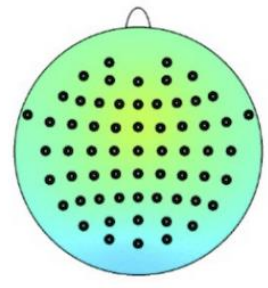

Congruent

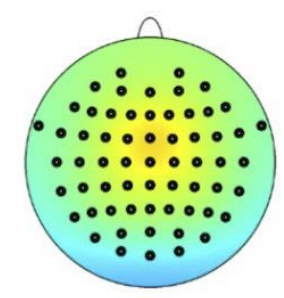

Incongruent

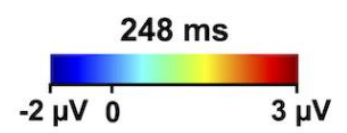

Figure 6. ERP amplitudes time-locked to the onset of the head cue: The graph illustrates Event-Related Spectral Perturbation (ERSP) amplitudes at $\mathrm{FCz}$ that were time-locked to the robot's head cue (A). Analysis of the ERSP amplitudes showed larger frontal theta amplitudes for incongruent vs. congruent signals (B). The graph also illustrates the topographies for each condition, which suggests the mid-frontal origin of theta frequencies (C).

\section{Discussion}

Experiment 3 aimed to examine the electrophysiological correlates of cognitive conflict that arises when viewing social signals incongruent with the task goal. 
Participants completed an identical task as in Study 1 and 2. The behavioral results of Study 3 replicated behavioral effects of Study 1 and 2 by showing that participants were faster to respond when the robot looked congruently at the correct target bin, compared to when it looked in the opposite direction of the correct target bin. However, the ambiguity of the object did not further modulate this effect. However, the error rates do show a similar effect as Study 1 where participants were more likely to make errors when the robot looked at the incongruent location and when the object was more ambiguous (i.e., the $75 \%$ objects). The ERP analyses showed that N2 amplitudes were more negative when the robot signal was incongruent. This effect was more pronounced when the object was not ambiguous (i.e., the $100 \%$ object), which is not completely in line with the behavioral data (i.e., the difference in error rates between congruent and incongruent trials was larger for the $75 \%$ objects). One possible explanation for this discrepancy could be that the N2 activity indexes general conflict monitoring. The larger conflict was elicited by the gaze shift directed to the wrong bin, observed in larger negative deflections in that condition. However, in the congruent condition, when the social signal corresponds to the observers' expectations, the differences in N2 might be related to the object itself: amplitude for the $100 \%$ object is higher than the $75 \%$ object. The object ambiguity (detected at the level of processing of the N2 component) might then be reflected in the error rate patterns, with higher differences in error rates for ambiguous objects relative to $100 \%$ yellow or blue objects. This interpretation would align with previous research that suggests that $\mathrm{N} 2$ is sensitive to the degree of perceptual deviation between stimuli (Folstein \& Van Petten, 2008).

Time-frequency (TF) analysis of the data showed a pattern in line with behavioral measures, namely that viewing incongruent head movements elicited higher conflict- 
related theta amplitudes than viewing congruent movements. Unlike the N2, the ERSP analysis did not show an effect of object type on theta amplitudes. This could be due to the fact that the $\mathrm{N} 2$ and frontal theta correspond to related but dissimilar cognitive processes (Cavanagh \& Frank, 2014) (Cavanaugh et al., 2010). For instance, while the N2 has been established as an index of violation of expectations, the frontal theta could reflect conflict processes.

\section{General Discussion}

In three Studies, we aimed to investigate the impact of irrelevant or incongruent social signals on cognitive control. Specifically, we evaluated the impact of conflicting robot social signals on performance reflected in behavioral and neural markers during categorization task requiring cognitive control. We asked participants to complete a simulated handover task in which they had to categorize an object that a robot "handed" to them based on its color by choosing the corresponding bin. After handing the object over, the robot either looked congruently at the correct bin (e.g., the object was blue, and the robot looked at the blue bin) or incongruently at the incorrect bin (e.g., the object was blue and the robot looked at the yellow bin).

We hypothesized that viewing incongruent head movements would be associated with behavioral and electrophysiological indices of cognitive conflict. As such, we expected longer RTs and more error rates in Study 1 for incongruent head signals compared to congruent head signals. For Study 2, we expected longer saccadic RTs and different gaze-path trajectories for incongruent signals compared to congruent signals. For Study 3, we expected larger N2 and frontal-theta amplitudes for incongruent signals vs. congruent head movements. 
In line with our hypotheses, the results of the three studies showed longer reaction times for incongruent head movements of the iCub robot compared to congruent head movements (Study 1-3) and more errors for incongruent compared to congruent head movements (Study 1 and 3). This difference in error rates was even more prominent when participants viewed a more ambiguous object (Study 1 and 3, and a similar descriptive pattern in Study 2). The results also reveal that gaze trajectories differed depending on the congruency of the gaze signals, where larger saccadic curvatures were observed when participants were viewing incongruent head signals compared to congruent head signals (Study 2). The EEG study results revealed more negative N2 amplitudes for the incongruent condition (Study 3), with a larger difference between incongruent and congruent trials for the unambiguous object vs. the ambiguous object (Study 3). Finally, ERSP analyses of the EEG signal showed larger frontal-theta amplitudes for the incongruent vs. congruent head movements.

The results of these studies highlight a few main findings. First, the fact that reaction times for congruent head movements of the observed agent were consistently faster than incongruent signals is in line with the well-studied gaze-cueing effect, which has been shown to be a reflexive attentional process (Friesen \& Kingstone, 1998). Additionally, and more interestingly, our results demonstrate that incongruent social signals from an artificial agent-induced conflict during the simulation of a handover task. This suggests that participants could engage in cognitive control, which involves inhibiting a distractor stimulus (Botvinick et al., 2004; Yeung et al., 2004; i.e., in our case, it is an incongruent robot signal) while completing a categorization task with a robotic agent. Additionally, our results demonstrate an unexpected finding that replicated across all three experiments, which dissociates between reaction time data 
and error rates. Namely, while both indices illustrated that processing was less efficient for incongruent signals, error rates were susceptible to the type of object that the participants viewed (i.e., ambiguous vs. unambiguous object). Reaction time data, however, were agnostic to the type of object that participants saw. This discrepancy suggests that the two variables could have different underlying cognitive mechanisms behind them. For example, reaction time data could be a better index for attentional processes while error rates could reflect cognitive conflict processing.

Since we did not expect the differences between reaction time and error rate data, this study cannot entirely address why this discrepancy is evident. However, existing literature can shed some light on these differences. One explanation could be that the cognitive load induced by the object's ambiguity influenced attentional processes and cognitive control differently. Specifically, studies have shown that increased cognitive load causes reflexive processes to take over in attentional processing, which would drive responses to a signal's directionality regardless of the goal of the task (Abubshait et al., 2020). On the other hand, studies examining cognitive conflict have shown that cognitive load is linked to enhanced conflict monitoring, allowing people to monitor further their behavior (Amodio et al., 2008). When we explain our findings within this context, we can reconcile the differences between reaction time findings and error rates. The fact that only congruency of the head movement with the target bin affected RTs could be due to the fact that the robots' signals would reflexively prompt a location, regardless of where the object should go, and as such, shows reduced RTs for congruent signals, but no effect of the object type. Simultaneously, we postulate that error rate differences related to congruency and object type may reflect how cognitive control mechanisms inhibits irrelevant stimuli (i.e., incongruent head movement) to achieve the 
goal at hand (i.e., selecting the correct bin). Importantly, we found that ambiguous objects elicited more error rates, which might be the result of higher cognitive load.

The object's ambiguity was also reflected in the amplitude of N2 in the congruent condition (no conflict related to the social signal), which was more negative for the ambiguous object, relative to the unambiguous object. This is also supported by studies that illustrate how the $\mathrm{N} 2$ and mid-line theta oscillations are related to different cognitive processes (Cohen \& Donner, 2013). Another alternative explanation could be that both RTs and error rates index conflict processing, but the error rates might be more sensitive to detect variations in the object ambiguity. However, the present results cannot distinguish between these alternative interpretations. Future studies should examine the discrepancy between RTs and error rates in relation to cognitive load and cognitive control in the presence of (irrelevant) social signals.

Our results also add to the body of literature that suggests that the N2 and frontal-theta oscillations are related components but do not reflect identical cognitive processes even though they share similar timing and scalp topographies (Cavanagh \& Frank, 2014). In the present studies, we showed that the N2 was sensitive to both congruent signals and an object's ambiguity. However, theta oscillations were only sensitive to the congruency of the signal regardless of the object type. On the other hand, the N2 was modulated due to the fact that it is a component observed during perceptual mismatch and not only prediction error (Folstein \& Van Petten, 2008).

Furthermore, the fact that all dependent variables (error rates, the N2 amplitudes, and frontal-theta amplitudes) showed consistent patterns suggests that cognitive conflict experienced in our paradigm is a highly stable effect (Cavanagh \& Frank, 2014; Cohen, 2014; Cohen \& Donner, 2013; Nigbur et al., 2011). Specifically, 
cognitive conflict arises because incongruent signals activate two different responses (i.e., the correct response and the response that corresponds to where the robot is looking) when only one should be selected (Cohen, 2014). In line with our interpretation, studies have shown that in tasks where two responses are activated and only one should be chosen, cognitive conflict arises (e.g., the Stroop task; Leuthold, 2011; Stroop, 1935 and the Go/NoGo tasks; Nigbur et al., 2011). During congruent trials, response selection is reasonably straightforward. However, "incongruent" or "NoGo" trials induce interference, which increases the cognitive demand, which is followed by cognitive conflict measured via behavioral (e.g., longer RTs, more errors) and neural markers (e.g., frontal-theta and N2; Cohen, 2014; Nigbur et al., 2011; Cavanagh \& Frank, 2014; Cohen \& Donner, 2013). Interestingly, the present study shows that social signals from an artificial agent in a handover simulation scenario can also induce cognitive conflict despite being irrelevant to the task at hand.

While it cannot be ruled out that non-social incongruent stimuli (e.g., arrows) can similarly exert a cognitive conflict (Gregory \& Jackson, 2017; Gregory \& Jackson, 2019; Marotta et al., 2012; Marotta et al., 2018; Marotta et al., 2019), the aim of this study was to examine whether humanoid robots/artificial agents, can exert cognitive conflict through their behavior. Although it remains to be answered whether these specific findings would also be observed in physical human-robot interaction (as opposed to screen-based experiments), it is entirely plausible since previous work has shown that psychological phenomena from screen-based experiments extend to physical human-robot interaction (Kompatsiari et al., 2018; Abubshait \& Wykowska, 2020).

With this in mind, one can conclude, based on our results, that endowing artificial agents with faces, eyes and social behavior might be counterproductive in 
situations that require humans to complete collaborative tasks. However, in other contexts (e.g., elderly care), social signals might be desirable. Thus, endowing artificial agents with social signals might depend on the area of application. Interdisciplinary work between cognitive neuroscientists and engineers should aim to create robots, virtual agents and avatars that carefully deliver social signals where needed, while preventing "social signal leaking" (i.e., undesired and distracting social signals such as facial expressions, eye movements or head shifts).

Furthermore, it is crucial to understand the consequences of cognitive conflict on performance during collaborative tasks. However, it is still unclear if the cognitive conflict would reduce the desirability of interaction, which could negatively affect the usage of robots. Additionally, measuring cognitive conflict is vital for human-robot interaction as it allows us to design more dynamic robots by incorporating fast neurophysiological measures online such as EEG in brain-computer interfaces (BCI).

\section{Conclusion}

The present study shows that the effect of irrelevant social signals from a humanoid robot on performance and cognition during goal-oriented tasks can be reliably indexed by behavioral measures, eye movements and electrophysiological markers associated with cognitive conflict. This shows that cognitive control mechanisms intervene during the observation of incongruent social signals to successfully achieve goals in a collaborative scenario with a humanoid robot. Our results allow us to speculate that similar attentional/cognitive monitoring mechanisms would occur during human-human interaction, as we argue that embodied humanoid robots are a proxy for social inter-personal interaction (see Wykowska, 2020 for review). However, whether the findings indeed do generalize to social interactions with other 
humans remain to be examined in future research. It is also of interest for future research whether the cognitive mechanisms elicited by the robot's social signals are social or of general purpose.

\section{Acknowledgments}

The authors would like to acknowledge Dr. Cesco Willemse for his help in analyzing and interpreting the eye-tracking data.

\section{Funding}

This work was supported by the European Research Council (ERC) under the European Union's Horizon 2020 research and innovation program, ERC Starting Grant ERC2016-StG-715058, awarded to AW, titled “InStance: Intentional Stance for Social Attunement”. The content of this paper is the sole responsibility of the authors. The European Commission or its services cannot be held responsible for any use that may be made of the information it contains.

\section{Contribution}

JPO, AA and AW conceptualized and designed the experiment. JPO and AA programmed the experiment. JPO and AA collected and analyzed the data. JPO, AA and AW interpreted the data and wrote the manuscript.

\section{References}

Abubshait, A., Momen, A., \& Wiese, E. (2020). Pre-exposure to Ambiguous Faces Modulates Top-Down Control of Attentional Orienting to Counterpredictive Gaze Cues. Frontiers in Psychology, 11. https://doi.org/10.3389/fpsyg.2020.02234

Abubshait, A., \& Wiese, E. (2017). You look human, but act like a machine: Agent appearance and behavior modulate different aspects of human-robot interaction. Frontiers in Psychology, 8. https://doi.org/10.3389/fpsyg.2017.01393

Abubshait, A., \& Wykowska, A. (2020). Repetitive Robot Behavior Impacts Perception of Intentionality and Gaze-Related Attentional Orienting. Frontiers in Robotics and AI, 7. https://doi.org/10.3389/frobt.2020.565825 
Manuscript accepted for publication in the Journal of Cognitive Neuroscience - JoCN

doi: 10.1162/jocn_a_o1786

Adolphs, R. (2003). Cognitive neuroscience of human social behaviour: Cognitive neuroscience. Nature Reviews Neuroscience, 4(3), 165-178. https://doi.org/10.1038/nrn1056

Amodio, D. M., Master, S. L., Yee, C. M., \& Taylor, S. E. (2008). Neurocognitive components of the behavioral inhibition and activation systems: Implications for theories of self-regulation. Psychophysiology, 45(1), 11-19. https://doi.org/10.1111/j.1469-8986.2007.00609.x

Bobak, A. K., \& Langton, S. R. H. (2015). Working memory load disrupts gaze-cued orienting of attention. Frontiers in Psychology, 6. https://doi.org/10.3389/fpsyg.2015.01258

Bocquillon, P., Bourriez, J.-L., Palmero-Soler, E., Molaee-Ardekani, B., Derambure, P., \& Dujardin, K. (2014). The spatiotemporal dynamics of early attention processes: A highresolution electroencephalographic study of N2 subcomponent sources. Neuroscience, 271, 922. https://doi.org/10.1016/j.neuroscience.2014.04.014

Bonifacci, P., Ricciardelli, P., Lugli, L., \& Pellicano, A. (2008). Emotional attention: Effects of emotion and gaze direction on overt orienting of visual attention. Cognitive Processing, 9(2), 127-135. https://doi.org/10.1007/s10339-007-0198-3

Botvinick, M. M., Braver, T. S., Barch, D. M., Carter, C. S., \& Cohen, J. D. (2001). Conflict monitoring and cognitive control. Psychological Review, 108(3), 624-652. https://doi.org/10.1037/0033-295X.108.3.624

Botvinick, M. M., Cohen, J. D., \& Carter, C. S. (2004). Conflict monitoring and anterior cingulate cortex: An update. Trends in Cognitive Sciences, 8(12), 539-546. https://doi.org/10.1016/j.tics.2004.10.003

Boucher, J.-D., Pattacini, U., Lelong, A., Bailly, G., Elisei, F., Fagel, S., Dominey, P., \& VentreDominey, J. (2012). I Reach Faster When I See You Look: Gaze Effects in Human-Human and Human-Robot Face-to-Face Cooperation. Frontiers in Neurorobotics, 6, 3 . https://doi.org/10.3389/fnbot.2012.00003

Brignani, D., Guzzon, D., Marzi, C. A., \& Miniussi, C. (2009). Attentional orienting induced by arrows and eye-gaze compared with an endogenous cue. Neuropsychologia, 47(2), 370-381. https://doi.org/10.1016/j.neuropsychologia.2008.09.011

Callejas, A., Shulman, G. L., \& Corbetta, M. (2014). Dorsal and Ventral Attention Systems Underlie Social and Symbolic Cueing. Journal of Cognitive Neuroscience, 26(1), 63-80. https://doi.org/10.1162/jocn_a_00461

Capozzi, F., \& Ristic, J. (2020). Attention AND mentalizing? Reframing a debate on social orienting of attention. Visual Cognition, 1-9. https://doi.org/10.1080/13506285.2020.1725206

Cavanagh, J. F., Cohen, M. X., \& Allen, J. J. B. (2009). Prelude to and Resolution of an Error: EEG Phase Synchrony Reveals Cognitive Control Dynamics during Action Monitoring. Journal of Neuroscience, 29(1), 98-105. https://doi.org/10.1523/JNEUROSCI.4137-08.2009

Cavanagh, J. F., \& Frank, M. J. (2014). Frontal theta as a mechanism for cognitive control. Trends in Cognitive Sciences, 18(8), 414-421. https://doi.org/10.1016/j.tics.2014.04.012

Cavanagh, J. F., Zambrano-Vazquez, L., \& Allen, J. J. B. (2012). Theta lingua franca: A common mid-frontal substrate for action monitoring processes. Psychophysiology, 49(2), 220-238. https://doi.org/10.1111/j.1469-8986.2011.01293.x 
Cazzato, V., Liuzza, M. T., Caprara, G. V., Macaluso, E., \& Aglioti, S. M. (2015). The attracting power of the gaze of politicians is modulated by the personality and ideological attitude of their voters: A functional magnetic resonance imaging study. European Journal of Neuroscience, 42(8), 2534-2545. https://doi.org/10.1111/ejn.13038

Chaminade, T., \& Okka, M. M. (2013). Comparing the effect of humanoid and human face for the spatial orientation of attention. Frontiers in Neurorobotics, 7. https://doi.org/10.3389/fnbot.2013.00012

Chevalier, P., Kompatsiari, K., Ciardo, F., \& Wykowska, A. (2020). Examining joint attention with the use of humanoid robots-A new approach to study fundamental mechanisms of social cognition. Psychonomic Bulletin \& Review, 27(2), 217-236. https://doi.org/10.3758/s13423019-01689-4

Ciardo, F., Marino, B. F. M., Actis-Grosso, R., Rossetti, A., \& Ricciardelli, P. (2014). Face age modulates gaze following in young adults. Scientific Reports, 4, 4746. https://doi.org/10.1038/srepo4746

Clayson, P. E., Carbine, K. A., Baldwin, S. A., \& Larson, M. J. (2019). Methodological reporting behavior, sample sizes, and statistical power in studies of event-related potentials: Barriers to reproducibility and replicability. Psychophysiology, 56(11), e13437. https://doi.org/10.1111/psyp.13437

Cohen, J. (1992). Statistical Power Analysis. Current Directions in Psychological Science, 1(3), 98-101. https://doi.org/10.1111/1467-8721.ep10768783

Cohen, M. X. (2014). A neural microcircuit for cognitive conflict detection and signaling. Trends in Neurosciences, 37(9), 480-490. https://doi.org/10.1016/j.tins.2014.06.004

Cohen, M. X., \& Cavanagh, J. F. (2011). Single-Trial Regression Elucidates the Role of Prefrontal Theta Oscillations in Response Conflict. Frontiers in Psychology, 2. https://doi.org/10.3389/fpsyg.2011.00030

Cohen, M. X., \& Donner, T. H. (2013). Midfrontal conflict-related theta-band power reflects neural oscillations that predict behavior. Journal of Neurophysiology, 110(12), 2752-2763. https://doi.org/10.1152/jn.00479.2013

Cole, G. G., Smith, D. T., \& Atkinson, M. A. (2015). Mental state attribution and the gaze cueing effect. Attention, Perception, \& Psychophysics, 77(4), 1105-1115. https://doi.org/10.3758/s13414-014-0780-6

Dalmaso, M., Castelli, L., \& Galfano, G. (2020a). Social modulators of gaze-mediated orienting of attention: A review. Psychonomic Bulletin \& Review. https://doi.org/10.3758/s13423-02001730-x

Dalmaso, M., Castelli, L., \& Galfano, G. (2020b). Early saccade planning cannot override oculomotor interference elicited by gaze and arrow distractors. Psychonomic Bulletin \& Review, 27(5), 990-997. https://doi.org/10.3758/s13423-020-01768-x

Dalmaso, M., Galfano, G., \& Castelli, L. (2015). The Impact of Same- and Other-Race Gaze Distractors on the Control of Saccadic Eye Movements. Perception, 44(8-9), 1020-1028. https://doi.org/10.1177/0301006615594936 
Manuscript accepted for publication in the Journal of Cognitive Neuroscience - JoCN

doi: 10.1162/jocn_a_o1786

Eriksen, B. A., \& Eriksen, C. W. (1974). Effects of noise letters upon the identification of a target letter in a nonsearch task. Perception \& Psychophysics, 16(1), 143-149. https://doi.org/10.3758/BFo3203267

Folstein, J. R., \& Van Petten, C. (2008). Influence of cognitive control and mismatch on the N2 component of the ERP: A review. Psychophysiology, 45(1), 152-170. https://doi.org/10.1111/j.1469-8986.2007.00602.x

Fox, E., Mathews, A., Calder, A. J., \& Yiend, J. (2007). Anxiety and sensitivity to gaze direction in emotionally expressive faces. Emotion, 7(3), 478-486. https://doi.org/10.1037/1528$3542 \cdot 7 \cdot 3 \cdot 478$

Friesen, C. K., \& Kingstone, A. (1998). The eyes have it! Reflexive orienting is triggered by nonpredictive gaze. Psychonomic Bulletin \& Review, 5(3), 490-495.

Frischen, A., Bayliss, A. P., \& Tipper, S. P. (2007). Gaze cueing of attention: Visual attention, social cognition, and individual differences. Psychological Bulletin, 133(4), 694-724. https://doi.org/10.1037/0033-2909.133.4.694

Gallagher, H. L., \& Frith, C. D. (2003). Functional imaging of 'theory of mind.' Trends in Cognitive Sciences, 7(2), 77-83. https://doi.org/10.1016/S1364-6613(02)00025-6

Gobel, M. S., Tufft, M. R. A., \& Richardson, D. C. (2017). Social beliefs and visual attention: How the social relevance of a cue influences spatial orienting. Cognitive Science, 42, 161-185. https://doi.org/10.1111/cogs.12529

Graham, R., Kelland Friesen, C., Fichtenholtz, H. M., \& LaBar, K. S. (2010). Modulation of reflexive orienting to gaze direction by facial expressions. Visual Cognition, 18(3), 331-368. https://doi.org/10.1080/13506280802689281

Gregory, S. E. A., \& Jackson, M. C. (2017). Joint attention enhances visual working memory. Journal of Experimental Psychology: Learning, Memory, and Cognition, 43(2), 237-249. https://doi.org/10.1037/xlmooo0294

Gregory, S. E. A., \& Jackson, M. C. (2019). Barriers block the effect of joint attention on working memory: Perspective taking matters. Journal of Experimental Psychology: Learning, Memory, and Cognition, 45(5), 795-806. https://doi.org/10.1037/xlmooo0622

Heil, M., Osman, A., Wiegelmann, J., Rolke, B., \& Hennighausen, E. (2000). N20o in the Eriksen-task: Inhibitory executive process? Journal of Psychophysiology, 14(4), 218-225. https://doi.org/10.1027/0269-8803.14.4.218

Hietanen, J. K. (2018). Affective Eye Contact: An Integrative Review. Frontiers in Psychology, 9, 1587. https://doi.org/10.3389/fpsyg.2018.01587

Huang, C.-M., \& Thomaz, A. L. (2011). Effects of responding to, initiating and ensuring joint attention in human-robot interaction. $2011 \quad R O-M A N, \quad 65-71$. https://doi.org/10.1109/ROMAN.2011.6005230

Hungr, C. J., \& Hunt, A. R. (2012). Physical self-similarity enhances the gaze-cueing effect. Quarterly Journal of Experimental Psychology, 65(7), 1250-1259. https://doi.org/10.1080/17470218.2012.690769 
Manuscript accepted for publication in the Journal of Cognitive Neuroscience - JoCN

doi: 10.1162/jocn_a_o1786

Ishikawa, M., Haensel, J. X., Smith, T. J., Senju, A., \& Itakura, S. (2021). Affective priming enhances gaze cueing effect. Journal of Experimental Psychology: Human Perception and Performance, 47(2), 189-199. https://doi.org/10.1037/xhpoooo880

Kawai, N. (2011). Attentional shift by eye gaze requires joint attention: Eye gaze cues are unique to shift attention1: Social attention by the gaze cues. Japanese Psychological Research, 53(3), 292-301. https://doi.org/10.1111/j.1468-5884.2011.00470.x

Kerns, J. G., Cohen, J. D., MacDonald, A. W., Cho, R. Y., Stenger, V. A., \& Carter, C. S. (2004). Anterior Cingulate Conflict Monitoring and Adjustments in Control. Science, 303(5660), 10231026. https://doi.org/10.1126/science.1089910

Kompatsiari, K., Ciardo, F., Tikhanoff, V., Metta, G., \& Wykowska, A. (2018). On the role of eye contact in gaze cueing. Scientific Reports, 8(1), 17842. https://doi.org/10.1038/s41598-01836136-2

Kompatsiari, K., Tikhanoff, V., Ciardo, F., Metta, G., \& Wykowska, A. (2017). The Importance of Mutual Gaze in Human-Robot Interaction. 2, 443-452. https://doi.org/10.1007/978-3-31970022-9_44

KOPP, B., RIST, F., \& MATTLER, U. (1996). N2Oo in the flanker task as a neurobehavioral tool for investigating executive control. Psychophysiology, 33(3), 282-294. https://doi.org/10.1111/j.1469-8986.1996.tbo0425.x

Kuhn, G., Benson, V., Fletcher-Watson, S., Kovshoff, H., McCormick, C. A., Kirkby, J., \& Leekam, S. R. (2010). Eye movements affirm: Automatic overt gaze and arrow cueing for typical adults and adults with autism spectrum disorder. Experimental Brain Research, 201(2), 155165. https://doi.org/10.1007/s00221-009-2019-7

Kuhn, G., Vacaityte, I., D’Souza, A. D. C., Millett, A. C., \& Cole, G. G. (2018). Mental states modulate gaze following, but not automatically. Cognition, 180, 1-9. https://doi.org/10.1016/j.cognition.2018.05.020

Leuthold, H. (2011). The Simon effect in cognitive electrophysiology: A short review. Acta Psychologica, 136(2), 203-211. https://doi.org/10.1016/j.actpsy.2010.08.001

Ludwig, C. J. H., \& Gilchrist, I. D. (2002). Measuring saccade curvature: A curve-fitting approach. Behavior Research Methods, Instruments, \& Computers, 34(4), 618-624. https://doi.org/10.3758/BFo3195490

Marotta, A., Lupiáñez, J., Martella, D., \& Casagrande, M. (2012). Eye gaze versus arrows as spatial cues: Two qualitatively different modes of attentional selection. Journal of Experimental Psychology: Human Perception and Performance, 38(2), 326-335. https://doi.org/10.1037/a0023959

Marotta, A., Lupiáñez, J., Román-Caballero, R., Narganes-Pineda, C., \& Martín-Arévalo, E. (2019). Are eyes special? Electrophysiological and behavioural evidence for a dissociation between eye-gaze and arrows attentional mechanisms. Neuropsychologia, 129, 146-152. https://doi.org/10.1016/j.neuropsychologia.2019.03.017

Marotta, A., Román-Caballero, R., \& Lupiáñez, J. (2018). Arrows don’t look at you: Qualitatively different attentional mechanisms triggered by gaze and arrows. Psychonomic Bulletin \& Review, 25(6), 2254-2259. https://doi.org/10.3758/s13423-018-1457-2 
Manuscript accepted for publication in the Journal of Cognitive Neuroscience - JoCN

doi: 10.1162/jocn_a_o1786

Mathôt, S., Schreij, D., \& Theeuwes, J. (2012). OpenSesame: An open-source, graphical experiment builder for the social sciences. Behavior Research Methods, 44(2), 314-324. https://doi.org/10.3758/s13428-011-0168-7

Metta, G., Natale, L., Nori, F., Sandini, G., Vernon, D., Fadiga, L., von Hofsten, C., Rosander, K., Lopes, M., Santos-Victor, J., Bernardino, A., \& Montesano, L. (2010). The iCub humanoid robot: An open-systems platform for research in cognitive development. Social Cognition: From Babies to Robots, 23(8), 1125-1134. https://doi.org/10.1016/j.neunet.2010.08.010

Mitchell, D. J., McNaughton, N., Flanagan, D., \& Kirk, I. J. (2008). Frontal-midline theta from the perspective of hippocampal "theta." Progress in Neurobiology, 86(3), 156-185. https://doi.org/10.1016/j.pneurobio.2008.09.005

Moore, R. A., Gale, A., Morris, P. H., \& Forrester, D. (2006). Theta phase locking across the neocortex reflects cortico-hippocampal recursive communication during goal conflict resolution. International Journal of Psychophysiology, 60(3), 260-273. https://doi.org/10.1016/j.ijpsycho.2005.06.003

Mutlu, B., Forlizzi, J., \& Hodgins, J. (2006). A storytelling robot: Modeling and evaluation of human-like gaze behavior. Humanoid. Robots. IEEE, 1-6. https://doi.org/10.1109/ICHR.2006.321322

Nieuwenhuis, S., Yeung, N., van den Wildenberg, W., \& Ridderinkhof, K. R. (2003). Electrophysiological correlates of anterior cingulate function in a go/no-go task: Effects of response conflict and trial type frequency. Cognitive, Affective, \& Behavioral Neuroscience, 3(1), 17-26. https://doi.org/10.3758/CABN.3.1.17

Nigbur, R., Ivanova, G., \& Stürmer, B. (2011). Theta power as a marker for cognitive interference. Clinical Neurophysiology, 2185-2194. https://doi.org/10.1016/j.clinph.2011.03.030

Palan, S., \& Schitter, C. (2018). Prolific.ac-A subject pool for online experiments. Journal of Behavioral and Experimental Finance, 17, 22-27. https://doi.org/10.1016/j.jbef.2017.12.004

Pecchinenda, A., \& Petrucci, M. (2016). Emotion Unchained: Facial Expression Modulates Gaze Cueing under Cognitive Load. PLOS ONE, 11(12), e0168111. https://doi.org/10.1371/journal.pone.0168111

Peirce, J., Gray, J. R., Simpson, S., MacAskill, M., Höchenberger, R., Sogo, H., Kastman, E., \& Lindeløv, J. K. (2019). PsychoPy2: Experiments in behavior made easy. Behavior Research Methods, 51(1), 195-203. https://doi.org/10.3758/s13428-018-01193-y

Perez-Osorio, J., Müller, H. J., Wiese, E., \& Wykowska, A. (2015). Gaze following is modulated by expectations regarding others' action goals. PLOS ONE, 1O(11), e0143614. https://doi.org/10.1371/journal.pone.0143614

Perez-Osorio, J., Müller, H. J., \& Wykowska, A. (2017). Expectations regarding action sequences modulate electrophysiological correlates of the gaze-cueing effect. Psychophysiology, 54(7), 942-954. https://doi.org/10.1111/psyp.12854

Porciello, G., Liuzza, M. T., Minio-Paluello, I., Caprara, G. V., \& Aglioti, S. M. (2016). Fortunes and misfortunes of political leaders reflected in the eyes of their electors. Experimental Brain Research, 234(3), 733-740. https://doi.org/10.1007/s00221-015-4496-1 
Manuscript accepted for publication in the Journal of Cognitive Neuroscience - JoCN

doi: 10.1162/jocn_a_o1786

Quadflieg, S., Mason, M. F., \& Macrae, C. N. (2004). The owl and the pussycat: Gaze cues and visuospatial orienting. Psychonomic Bulletin \& Review, 11(5), 826-831. https://doi.org/10.3758/BFo3196708

Ricciardelli, P., Bricolo, E., Aglioti, S. M., \& Chelazzi, L. (2002). My eyes want to look where your eyes are looking: Exploring the tendency to imitate another individual's gaze. NeuroReport, 13(17). https://journals.lww.com/neuroreport/Fulltext/2002/12030/My_eyes_want_to_look_where_ your_eyes_are_looking_.18.aspx

Sauseng, P., Griesmayr, B., Freunberger, R., \& Klimesch, W. (2010). Control mechanisms in working memory: A possible function of EEG theta oscillations. Binding Processes: Neurodynamics and Functional Role in Memory and Action, 34(7), 1015-1022. https://doi.org/10.1016/j.neubiorev.2009.12.006

Sauseng, P., Klimesch, W., Freunberger, R., Pecherstorfer, T., Hanslmayr, S., \& Doppelmayr, M. (2006). Relevance of EEG alpha and theta oscillations during task switching. Experimental Brain Research, 170(3), 295-301. https://doi.org/10.1007/s00221-005-0211-y

Simon, J. R., \& Wolf, j. D. (1963). Choice reaction time as a function of angular stimulusresponse correspondence and age. Ergonomics, 6(1), 99-105. https://doi.org/10.1080/00140136308930679

Stroop, J. R. (1935). Studies of interference in serial verbal reactions. Journal of Experimental Psychology, 18(6), 643-662. https://doi.org/10.1037/ho054651

Teufel, C., Fletcher, P. C., \& Davis, G. (2010). Seeing other minds: Attributed mental states influence perception. Trends in Cognitive Sciences, 14(8), 376-382. https://doi.org/10.1016/j.tics.2010.05.005

Tipples, J. (2006). Fear and fearfulness potentiate automatic orienting to eye gaze. Cognition and Emotion, 2O(2), 309-320. https://doi.org/10.1080/02699930500405550

Tudge, L., McSorley, E., Brandt, S. A., \& Schubert, T. (2017). Setting things straight: A comparison of measures of saccade trajectory deviation. Behavior Research Methods, 49(6), 2127-2145. https://doi.org/10.3758/s13428-016-0846-6

Tzur, G., \& Berger, A. (2007). When things look wrong: Theta activity in rule violation. Neuropsychologia, 45(13), https://doi.org/10.1016/j.neuropsychologia.2007.05.004

$3122-3126$.

Van der Stigchel, S., Meeter, M., \& Theeuwes, J. (2006). Eye movement trajectories and what they tell us. Neuroscience \& Biobehavioral Reviews, 30(5), 666-679. https://doi.org/10.1016/j.neubiorev.2005.12.001

Voytek, B., Kayser, A. S., Badre, D., Fegen, D., Chang, E. F., Crone, N. E., Parvizi, J., Knight, R. T., \& D'Esposito, M. (2015). Oscillatory dynamics coordinating human frontal networks in support of goal maintenance. Nature Neuroscience, 18(9), 1318-1324. https://doi.org/10.1038/nn.4071

Wiese, E., Buzzell, G. A., Abubshait, A., \& Beatty, P. J. (2018). Seeing minds in others: Mind perception modulates low-level social-cognitive performance and relates to ventromedial 
prefrontal structures. Cognitive, Affective, \& Behavioral Neuroscience, 18(5), 837-856. https://doi.org/10.3758/s13415-018-0608-2

Wiese, E., Mandell, A., Shaw, T., \& Smith, M. (2019). Implicit mind perception alters vigilance performance because of cognitive conflict processing. Journal of Experimental Psychology: Applied, 25(1), 25-40. https://doi.org/10.1037/xapoooo186

Wiese, E., Metta, G., \& Wykowska, A. (2017). Robots as intentional agents: Using neuroscientific methods to make robots appear more social. Frontiers in Psychology, 8. https://doi.org/10.3389/fpsyg.2017.01663

Wiese, E., Wykowska, A., Zwickel, J., \& Müller, H. J. (2012). I see what you mean: How attentional selection is shaped by ascribing intentions to others. PLOS ONE, 7(9), e45391. https://doi.org/10.1371/journal.pone.0045391

Wykowska, A. (2020). Social Robots to Test Flexibility of Human Social Cognition. International Journal of Social Robotics, 12(6), 1203-1211. https://doi.org/10.1007/s12369020-00674-5

Wykowska, A., Chaminade, T., \& Cheng, G. (2016). Embodied artificial agents for understanding human social cognition. Philosophical Transactions of the Royal Society of London. Series B, Biological Sciences, 371, 20150375. https://doi.org/10.1098/rstb.2015.0375

Wykowska, A., Wiese, E., Prosser, A., \& Müller, H. J. (2014). Beliefs about the minds of others influence how we process sensory information. PLoS ONE, 9(4), e94339. https://doi.org/10.1371/journal.pone.0094339

Yamanaka, K., \& Yamamoto, Y. (2009). Single-trial EEG Power and Phase Dynamics Associated with Voluntary Response Inhibition. Journal of Cognitive Neuroscience, 22(4), 714-727. https://doi.org/10.1162/jocn.2009.21258

Yeung, N. (2014). Conflict monitoring and cognitive control. In The Oxford handbook of cognitive neuroscience, Vol. 2: The cutting edges (pp. 275-299). Oxford University Press.

Yeung, N., Botvinick, M. M., \& Cohen, J. D. (2004). The Neural Basis of Error Detection: Conflict Monitoring and the Error-Related Negativity. Psychological Review, 111(4), 931-959. https://doi.org/10.1037/0033-295X.111.4.931

Zaki, J., Hennigan, K., Weber, J., \& Ochsner, K. N. (2010). Social Cognitive Conflict Resolution: Contributions of Domain-General and Domain-Specific Neural Systems. Journal of Neuroscience, 30(25), 8481-8488. https://doi.org/10.1523/JNEUROSCI.0382-10.2010 